Proceedings of the Royal Society of Edinburgh: Section A Mathematics

http://journals.cambridge.org/PRM

THE ROYAL SOCIETYOF

BDINBURGH

Additional services for Proceedings of the Royal Society of Edinburgh:

Section A Mathematics:

Email alerts: $\underline{\text { Click here }}$

Subscriptions: $\underline{\text { Click here }}$

Commercial reprints: $\underline{\text { Click here }}$

Terms of use : $\underline{\text { Click here }}$

\title{
An asymptotic formula for the voltage potential in a perturbed $\varepsilon$-periodic composite medium containing misplaced inclusions of size $\varepsilon$
}

M. F. Ben Hassen and E. Bonnetier

Proceedings of the Royal Society of Edinburgh: Section A Mathematics / Volume 136 / Issue 04 / August 2006, pp 669 - 700 DOI: 10.1017/S0308210500004650, Published online: 12 July 2007

Link to this article: http://journals.cambridge.org/abstract S0308210500004650

How to cite this article:

M. F. Ben Hassen and E. Bonnetier (2006). An asymptotic formula for the voltage potential in a perturbed $\varepsilon$-periodic composite medium containing misplaced inclusions of size $\varepsilon$. Proceedings of the Royal Society of Edinburgh: Section A Mathematics, 136, pp 669-700 doi:10.1017/S0308210500004650

Request Permissions : $\underline{\text { Click here }}$ 


\title{
An asymptotic formula for the voltage potential in a perturbed $\varepsilon$-periodic composite medium containing misplaced inclusions of size $\varepsilon$
}

\author{
M. F. Ben Hassen \\ Institut für Numerische und Angewandte Mathematik, \\ University of Göttingen, Lotzestrasse 16-18, 37083 Göttingen, \\ Germany (benhasse@math.uni-goettingen.de)

\section{E. Bonnetier} \\ LMC-IMAG, Université Joseph Fourier Grenoble, BP 53, \\ 38041 Grenoble Cedex 9, France (eric.bonnetier@imag.fr)
}

(MS received 24 February 2005; accepted 7 September 2005)

\begin{abstract}
We consider composite media made of homogeneous inclusions with $\mathcal{C}^{1, \alpha}$ boundaries. Our goal is to compare the potential $u_{\varepsilon}$ in a perfectly periodic composite with the potential $u_{\varepsilon, d}$ of a perturbed periodic medium, where the periodicity defects consist of misplaced inclusions. We give an asymptotic expansion of the difference $u_{\varepsilon, d}-u_{\varepsilon}$ away from the defects and show that, to first order, a misplaced inclusion manifests itself via a polarization tensor, which is characterized.
\end{abstract}

\section{Introduction}

In this work, we consider a composite medium made of an array of inclusions embedded in a homogeneous background material. We assume that the inclusions are centred on an $\varepsilon$-periodic lattice, except for a small number of them that may have been misplaced: the centres of these 'defects' are at a distance of order $\varepsilon$ from the lattice points. Our goal is to compare, sufficiently far from the defects, the potential $u_{\varepsilon, d}$ of the perturbed medium with the potential $u_{\varepsilon}$ of a perfectly periodic medium.

Fengya et al. [10] (see also [2] and the references therein) studied the perturbations of the potential caused by the presence of small inhomogeneities when the reference or background medium is homogeneous (or sufficiently smooth), and derived an asymptotic expansion for the difference between the perturbed and background potentials. The first correction term in their asymptotic expansion is of the order of the volume $\varepsilon^{n}$ of the inhomogeneities and has the following structure:

$$
\varepsilon^{n} \sum_{j=1}^{m} \nabla_{x} u\left(z_{j}\right) \cdot M_{j} \nabla_{x} G\left(z_{j}, z\right),
$$

where $u$ is the background potential and $M_{j}$ is a polarization tensor, which characterizes how the presence of the $j$ th inhomogeneity, centred at $z_{j}$, is felt in the far field. The above expression also involves the gradient of the background Green 
function $G(x, z)$, which makes the expansion particularly interesting for numerical detection of inhomogeneities: linear sampling or MUSIC algorithms detect the singularities of the Green functions, and have proven quite efficient in both impedance imaging of inhomogeneities of low volume fraction [7] and inverse scattering by small inclusions [3].

In this paper we derive a similar asymptotic formula when the background medium is periodic. Our main result, theorem 4.1, shows that inhomogeneities or defects similar in size to the period affect the perturbed potential in a manner similar to the case of a homogeneous background. Indeed, the first term in our asymptotic formula (4.4) has the same structure as (1.1). It involves the gradient of the homogenized potential at the centre of the inclusions, the gradient of the homogenized Green function, and a polarization tensor that combines the influence of the defect at infinity and the interaction of the defect with the periodic structure. Thus, numerical detection of such periodicity defects should be possible using Music algorithms, provided that one has accurate knowledge of the background potential (which might be expensive in practice).

A possible application of our analysis concerns photonic crystals, periodic composite arrays of dielectric materials. In these structures, propagation of waves may be prohibited in certain intervals of frequencies, as a result of destructive interferences between the waves and the structure of the composite [13]. For a mathematical perspective, see the enthusiastic review by Kuchment [14].

Photonic crystals are an example of structures where periodicity or near-periodicity seems to play an important role. As the current manufacturing processes may not guarantee perfect periodicity, it is interesting to study the influence of periodicity defects in these structures, in view of developing methods for non-destructive control.

Our analysis relies on fine regularity results on the potential gradients $[15,16]$, which require that the inclusions be somewhat smooth: their boundaries have regularity $\mathcal{C}^{1, \alpha}$ for some $0<\alpha \leqslant 1$. Under this hypothesis, $\left\|\nabla u_{\varepsilon, d}\right\|_{\infty}$ can be shown to be bounded independently of $\varepsilon$ and of the distance between inclusions, misplaced or not.

The paper is organized as follows. In $\S 2$, we recall classical results about Green functions for uniformly elliptic operators in divergence form with merely bounded and measurable coefficients. We are particularly interested in their behaviour at infinity, and throughout the paper we work in dimension 3 (although some of our results are valid and given in any dimension).

Section 3 is concerned only with the background potential $u_{\varepsilon}$. In $\S 3.1$, we recall a few classical results of periodic homogenization, while $\S 3.2$ contains several estimates on the potential $u_{\varepsilon}$ and on its gradient. We recall such interior estimates, which were derived by Avellaneda and Lin [4], when the coefficients of the medium have regularity $\mathcal{C}^{0, \mu}$. We then give interior estimates on the gradient $\nabla u_{\varepsilon}$, in the case of a composite medium made of a homogeneous background conductor containing homogeneous inclusions with $\mathcal{C}^{1, \alpha}$ boundaries (in which case the conductivity is only globally $L^{\infty}$ ). These estimates are similar to those given in [15] (and so is their proof) but here we allow a non-zero source term.

We chose to study the case of Neumann boundary conditions for the periodic and perturbed media. The analysis also led us to compare the potentials $u_{\varepsilon, d}$ and $u_{\varepsilon}$ with 
the potential $u_{0}$ of the homogenized medium. In particular, we give an $L^{2}$ error estimate on $u_{\varepsilon}-u_{0}$. This kind of estimate is well known in the case of Dirichlet boundary conditions. In the case of Neumann boundary conditions, our result generalizes to dimension 3, a two-dimensional estimate obtained by Moskow and Vogelius [20]. The proofs of all the estimates in this section are given in the appendix.

Section 4 contains the main result. We derive there the asymptotic expansion of the potential in the domain with defects. We give the expression of the polarization tensor associated with a periodicity defect and compare it with the formula of [10] that describes the effect at infinity of an inhomogeneity embedded in a smooth matrix.

Throughout the paper, $C$ denotes a generic positive constant, independent of $\varepsilon$.

\section{Properties of the Green function}

In this section, we present some known results and properties of the Green function for the elliptic operator

$$
L u=-\operatorname{div}(a(x) \nabla u),
$$

when the conductivity $a(x)$ is merely a bounded measurable function in $\mathbb{R}^{n}$. The detailed proofs of the following results can be found in $[18,21,22]$ in the symmetric case and are extended to the case of non-symmetric coefficients in [12].

Let $\Omega$ be a smooth bounded domain in $\mathbb{R}^{n}$. We consider a medium with conductivity $a \in L^{\infty}(\Omega)$ that is uniformly elliptic

$$
0<\lambda \leqslant a(x) \leqslant \Lambda \quad \text { a.e. } x \in \Omega \text {. }
$$

Given a Radon measure $\mu$ defined on $\Omega$, a function $u \in L^{1}(\Omega)$ is called a weak solution vanishing at the boundary $\partial \Omega$ of the equation $L u=\mu$, if it satisfies

$$
\int_{\Omega} u L \Phi \mathrm{d} x=\int_{\Omega} \Phi \mathrm{d} \mu
$$

for every $\Phi \in H_{0}^{1}(\Omega) \cap \mathcal{C}^{0}(\bar{\Omega})$ such that $L \Phi \in C^{0}(\bar{\Omega})$.

If $\mu=f$ with $f \in W^{-1,2}(\Omega)$, the Lax-Milgram lemma shows that there exists a unique solution $u \in H_{0}^{1}(\Omega)$ of

$$
L u=f .
$$

One can thus define a continuous linear operator $\mathcal{G}: W^{-1,2}(\Omega) \rightarrow H_{0}^{1}(\Omega)$, called the Green operator, such that, for $f \in W^{-1,2}(\Omega), u=\mathcal{G}(f)$ is the unique solution in $H_{0}^{1}(\Omega)$ of $L u=f$.

A theorem of Stampacchia [21] extends the De Giorgi-Nash theorem on $\mathcal{C}^{0, \alpha}$ regularity of solutions to elliptic equations and shows that, when $\Omega$ is sufficiently smooth and $f \in W^{-1, p}(\Omega)$ with $p>n$, the solution $u$ to $(2.2)$ lies in $\mathcal{C}^{0}(\bar{\Omega})$. Moreover, one has

$$
\forall f \in \mathcal{C}^{0}(\bar{\Omega}), \quad \max _{\bar{\Omega}}|\mathcal{G}(f)| \leqslant C \lambda|\Omega|^{1 / n-1 / p}\|f\|_{W^{-1, p}},
$$

where $C$ depends only on $p$. Consequently, given a Radon measure $\mu$, a function $u$ is a weak solution vanishing on $\partial \Omega$ of the equation $L u=\mu$ if and only if

$$
\forall f \in \mathcal{C}^{0}(\bar{\Omega}), \quad \int_{\Omega} u f \mathrm{~d} x=\int_{\Omega} \mathcal{G}(f) \mathrm{d} \mu .
$$


There is at most one solution to this problem. By (2.3), this solution satisfies

$$
\forall f \in \mathcal{C}^{0}(\bar{\Omega}), \quad \int_{\Omega} u f \mathrm{~d} x \leqslant C \lambda|\Omega|^{1 / n-1 / p} \int_{\Omega}|\mathrm{d} \mu|\|f\|_{W^{-1, p}(\Omega)} .
$$

Since $\mathcal{C}^{0}(\bar{\Omega})$ is dense in $W^{-1, p}(\Omega)$, we see that $u \in W_{0}^{1, p^{\prime}}(\Omega), 1 / p+1 / p^{\prime}=1 / n$, and

$$
\|u\|_{W_{0}^{1, p^{\prime}}(\Omega)} \leqslant C \lambda|\Omega|^{1 / p^{\prime}} \int_{\Omega}|\mathrm{d} \mu| .
$$

The transformation $\mu \rightarrow u$ is thus the adjoint operator $\mathcal{G}^{*}$ of $\mathcal{G}$ : as $\mathcal{G}\left(W^{-1, p}(\Omega)\right) \subset$ $\mathcal{C}^{0}(\Omega)$, the image by $\mathcal{G}^{*}$ of the space of Radon measures on $\Omega$ is contained in $W_{0}^{1, p^{\prime}}(\Omega)$. This proves the following theorem.

THEOREM 2.1 (Littman et al. [18]). There exists, for every Radon measure $\mu$, a unique weak solution $u$ to the equation $L u=\mu$ vanishing on $\partial \Omega$, which lies in $W_{0}^{1, q}(\Omega)$ for every $q<n /(n-1)$.

Moreover, $u$ belongs to $H_{0}^{1}(\Omega)$ if and only if $\mu \in W^{-1,2}(\Omega)$.

As a consequence, one can define a Green function for $L$ in $\Omega$, as follows.

Definition 2.2. The Green function $G(x, y)$, associated with the operator $L$ on $\Omega$, is defined as the weak solution vanishing on $\partial \Omega$ of the equation

$$
L G=\delta_{y} \quad \text { in } \Omega,
$$

where $\delta_{y}$ is the Dirac mass at $y$.

The Green function provides a representation formula (see [18, theorem 6.1]): for every Radon measure $\mu$, the integral

$$
u(x)=\int G(x, y) \mathrm{d} \mu(y)
$$

is finite almost everywhere (a.e.) and is the weak solution vanishing on $\partial \Omega$ of the equation $L u=\mu$.

The Green function has the following properties.

TheOREM 2.3 (Littman et al. [18]; Grüter and Widman [12]). For each $y \in \Omega$,

$$
\left.\begin{array}{rl}
G(\cdot, y) \in L_{n /(n-2)(\Omega)}^{*}, & \|G\|_{L_{n /(n-2)(\Omega)}^{*}} \leqslant C(n) \lambda^{-1}, \\
\nabla G(\cdot, y) \in L_{n /(n-1)(\Omega)}^{*}, \quad\|\nabla G\|_{L_{n /(n-2)(\Omega)}^{*}} \leqslant C(n, \lambda, \Lambda), \\
G(\cdot, y) \in W_{0}^{1, s}(\Omega) \quad \text { for } s \in\left[1, \frac{n}{n-1}[.\right.
\end{array}\right\}
$$

Further, let $G$ and $\bar{G}$ be the Green functions of two uniformly elliptic operators $L$ and $\bar{L}$, with ellipticity constants $\lambda, \Lambda$ and $\bar{\lambda}, \bar{\Lambda}$, respectively. Then for any compact subset $K \subset \subset \Omega$, there exist positive constants $c$ and $C$, which depend only on $K$, $\Omega, n$ and on the ellipticity constants, such that

$$
\forall(x, y) \in K \times K, \quad c \bar{G}(x, y) \leqslant G(x, y) \leqslant C \bar{G}(x, y) .
$$


The Lorentz spaces $L_{p}^{*}(\Omega)$ involved in these estimates are defined by

$$
L_{p}^{*}(\Omega):=\left\{f: \Omega \rightarrow \mathbb{R} \cup\{\infty\}, f \text { measurable and }\|f\|_{L_{p}^{*}(\Omega)}<\infty\right\},
$$

where

$$
\|f\|_{L_{p}^{*}(\Omega)}:=\sup _{t>0} t|\{x \in \Omega:|f(x)|>t\}|^{1 / p},
$$

and are related to the classical $L^{p}$ spaces via the estimates

$$
\left(\frac{p}{\beta}\right)^{1 /(p-\beta)}|\Omega|^{-\beta / p(p-\beta)}\|f\|_{L^{p-\beta}(\Omega)} \leqslant\|f\|_{L_{p}^{*}(\Omega)} \leqslant\|f\|_{L^{p}(\Omega)},
$$

for $0<\beta \leqslant p-1$.

When $n \geqslant 3$, as the radius of $\Omega$ tends to infinity, the Green function converges to a function $G(\cdot, y)$, Hölder continuous in $\mathbb{R}^{n} \backslash\{y\}$. Moreover, $G(\cdot, y) \in$ $W_{\text {loc }}^{1, q}\left(\mathbb{R}^{n}\right) \cap W_{\text {loc }}^{1,2}\left(\mathbb{R}^{n} \backslash y\right), q<n /(n-1)$, and the representation formula $(2.5)$ is valid. In particular, given $f \in W^{-1,2}\left(\mathbb{R}^{n}\right)$, the solution $u \in W^{1,2}\left(\mathbb{R}^{n}\right)$ of $L u=f$ can be represented by

$$
u(x)=\int_{\mathbb{R}^{n}} G(x, y) f(y) \mathrm{d} y .
$$

The estimates (2.7) hold uniformly in $\mathbb{R}^{n}$ with constants that depend only on the ellipticity constants and on $n$. Comparing the Green functions of $L$ and of the Laplace operator in $\mathbb{R}^{n}$, we see from (2.7) that

$$
\forall(x, y) \in \mathbb{R}^{n} \times \mathbb{R}^{n}, \quad|G(x, y)| \leqslant C|x-y|^{2-n},
$$

where $C$ depends only on $\lambda, \Lambda$ and $n$.

In the rest of this paper, we will be concerned with families of operators of the form $L_{\varepsilon}=\operatorname{div}(a(x / \varepsilon) \nabla)$ defined over a smooth domain $\Omega \subset \mathbb{R}^{3}$, where $a$ is a $[0,1]^{3}$-periodic piecewise constant function.

\section{Asymptotic behaviour of the background potential and of the associated Green function in periodic composite materials}

Let $\Omega$ be a smooth bounded domain in $\mathbb{R}^{3}$ that contains a periodic composite medium composed of cells of size $\varepsilon$. These cells are deduced from the unit cell $Y=(0,1)^{3}$ by translation and rescaling, and are of the form $\varepsilon p+\varepsilon Y, p \in \mathbb{Z}^{3}$. The unit cell $Y$ contains an inclusion $D_{0} \subset \subset Y$ with boundary of class $\mathcal{C}^{1, \alpha}, 0<\alpha \leqslant 1$. We assume that

$$
\operatorname{dist}\left(D_{0}, \partial Y\right) \geqslant d_{0}>0 .
$$

Let $0<\lambda \leqslant \Lambda$ and $0<\mu<1 . \mathcal{A}(\lambda, \Lambda, \mu, \alpha)$ denotes the class of $Y$-periodic functions $a$ such that $a$ is $\mathcal{C}^{0, \mu}$ in $\bar{D}_{0}$ and in $\overline{Y \backslash D_{0}}$ and such that $0<\lambda \leqslant a(x) \leqslant \Lambda$ in $Y$. We also denote by $\mathcal{L}(\lambda, \Lambda, \mu, \alpha)$ the class of elliptic operators with coefficients in $\mathcal{A}(\lambda, \Lambda, \mu, \alpha)$ of the form

$$
L_{\varepsilon}=-\operatorname{div}\left(a\left(\frac{x}{\varepsilon}\right) \nabla \cdot\right)=-\operatorname{div}\left(a_{\varepsilon}(x) \nabla \cdot\right), \quad 0<\varepsilon<1,
$$

where $a_{\varepsilon}(x)=a(x / \varepsilon)$. We call these media 'composites with sufficiently smooth inclusions'. 


\subsection{Homogenization}

As $\varepsilon$ tends to zero, we consider the sequence of elliptic problems

$$
\left.\begin{array}{rl}
L_{\varepsilon} u_{\varepsilon}=f & \text { in } \Omega, \\
a_{\varepsilon} \frac{\partial u_{\varepsilon}}{\partial \nu}=g & \text { on } \partial \Omega,
\end{array}\right\}
$$

with the normalization

$$
\int_{\partial \Omega} g \mathrm{~d} \sigma_{x}=\int_{\Omega} f \mathrm{~d} x \text { and } \int_{\Omega} u_{\varepsilon} \mathrm{d} x=0
$$

The effective behaviour of the composite and the asymptotic behaviour of $u_{\varepsilon}$ are described in terms of solutions $\chi \in\left(W_{\#}^{1,2}(Y)\right)^{3}$ and $\Phi \in\left(W_{\#}^{1,2}(Y)\right)^{3^{2}}$ to cell problems, defined by

$$
\begin{aligned}
-\operatorname{div}(a(y) \nabla(\chi(y)+y)) & =0 \quad \text { in } \mathbb{R}^{3}, & \int_{Y} \chi(y) \mathrm{d} y & =0, \\
-\operatorname{div}\left(a(y) \nabla_{y} \Phi(y)\right) & =B(y)-\int_{Y} B(y) \mathrm{d} y \quad \text { in } Y, & \int_{Y} \Phi(y) \mathrm{d} y & =0,
\end{aligned}
$$

with

$$
B(y)=a(y) I+a(y) \nabla_{y} \chi(y)+\operatorname{div}_{y}(a(y) I \otimes \chi(y))
$$

$\left(W_{\#}^{1,2}\right.$ denotes the subspace of periodic functions of $\left.W^{1,2}(Y)\right)$. The function $u_{\varepsilon}$ can be sought formally with the ansatz $[6,23]$

$$
u_{\varepsilon}(x)=u_{0}\left(x, \frac{x}{\varepsilon}\right)+\varepsilon u_{1}\left(x, \frac{x}{\varepsilon}\right)+\varepsilon^{2} u_{2}\left(x, \frac{x}{\varepsilon}\right)+\varepsilon^{3} u_{3}\left(x, \frac{x}{\varepsilon}\right)+\cdots,
$$

where each function $u_{i}(x, y)$ is $Y$-periodic with respect to the fast variable $y=x / \varepsilon$.

The function $u_{0}(x, y)=u_{0}(x)$ is independent from $y$ and is the unique solution in $W^{1,2}(\Omega)$ to the homogenized equation

$$
\left.\begin{array}{rl}
L_{0} u_{0} & =-\operatorname{div}\left(A \nabla u_{0}\right)=f \quad \text { in } \Omega, \\
\nabla u_{0} \cdot \nu & =g \quad \text { on } \partial \Omega,
\end{array}\right\}
$$

with the normalization

$$
\int_{\Omega} u_{0} \mathrm{~d} \sigma_{x}=0
$$

The effective properties of the medium are expressed by the constant, symmetric, positive definite, homogenized matrix $A$ defined by

$$
A=\int_{Y} a(y)(I+\nabla \chi(y)) \mathrm{d} y .
$$

The functions $u_{1}$ and $u_{2}$ can be written in terms of derivatives of $u_{0}$, up to arbitrary functions $\tilde{u}_{1}, \tilde{u}_{2}$ of the variable $x$ only:

$$
\begin{aligned}
& u_{1}\left(x, \frac{x}{\varepsilon}\right)=\chi\left(\frac{x}{\varepsilon}\right) \cdot \nabla u_{0}(x)+\tilde{u}_{1}(x), \\
& u_{2}\left(x, \frac{x}{\varepsilon}\right)=\Phi\left(\frac{x}{\varepsilon}\right): \nabla u_{0}(x)+\chi\left(\frac{x}{\varepsilon}\right) \cdot \nabla \tilde{u}_{1}(x)+\tilde{u}_{2}(x) .
\end{aligned}
$$


If we approximate $u_{\varepsilon}$ to first order by (3.7), we may choose $\tilde{u}_{1}=0$. If we seek an approximation up to second order away from the boundary (neglecting boundary layers) $\tilde{u}_{2}$ may be chosen to be 0 , but $\tilde{u}_{1}$ must satisfy

$$
-\operatorname{div}\left(A \nabla \tilde{u}_{1}\right)=C_{0} \nabla^{3} u_{0},
$$

where

$$
C_{0}=\int_{Y}(a(y) \nabla \Phi(y)+a(y) I \otimes \chi(y)) \mathrm{d} y
$$

\subsection{Error estimates}

In this section, we give $W^{1, \infty}$-interior estimates for solutions $u_{\varepsilon}$ to

$$
L_{\varepsilon} u_{\varepsilon}=f \quad \text { in } \Omega,
$$

i.e. we are concerned only with perfectly periodic media. We are particularly interested in pointwise estimates on the gradients of $u_{\varepsilon}$, which will be used in the proofs in $\S 4$.

When the conductivity $a$ has global Hölder regularity on $Y,\|a\|_{\mathcal{C}^{0, \mu}(Y)} \leqslant M$, Avellaneda and Lin [4] proved that the potentials $u_{\varepsilon}$ are uniformly Lipschitz.

Theorem 3.1 (Avellaneda and Lin [4, theorem 2]). Let $u_{\varepsilon}$ satisfy

$$
\begin{aligned}
L_{\varepsilon} u_{\varepsilon}=f & & \text { in } \Omega \subset \mathbb{R}^{n}, \\
u_{\varepsilon}=g & & \text { on } \partial \Omega,
\end{aligned}
$$

where $f \in L^{n+\delta}$ for some $\delta>0$ and $g \in \mathcal{C}^{1, \nu}(\partial \Omega), 0<\nu \leqslant 1$. There exists a constant $C$ that depends only on $\lambda, \Lambda, \mu M, \Omega, \nu$ and $\delta$, such that

$$
\left\|u_{\varepsilon}\right\|_{\mathcal{C}^{0,1}(\Omega)} \leqslant C\left(\|g\|_{\mathcal{C}^{1, \nu}(\partial \Omega)}+\|f\|_{L^{n+\delta}(\Omega)}\right) .
$$

The regularity hypothesis on $a$ can be relaxed to cover the case of composite media that contain inclusions with sufficiently smooth boundaries. For such media, one can show that the gradient of the potential is uniformly bounded, independently of the inter-inclusion distance. Results of this nature were first obtained by $\mathrm{Li}$ and Vogelius [16], and then generalized to strongly elliptic systems by Li and Nirenberg [15]. We state here the version of [15] in the scalar case.

Let $D$ be a bounded domain in $\mathbb{R}^{3}$ containing $L$ disjoint subdomains $D_{1}, \ldots, D_{L}$, of class $\mathcal{C}^{1, \alpha}, 0<\alpha \leqslant 1$, with $D=\left(\bigcup_{l=1}^{L} \bar{D}_{l}\right) \backslash \partial D$. We assume that any point $x \in D$ belongs to at most two of the boundaries of the $D_{l}$. For $\eta>0$, we set

$$
D_{\eta}=\{x \in D ; \operatorname{dist}(x, \partial D)>\eta\} .
$$

Theorem 3.2 (Li and Nirenberg [15, theorem 0.1]). Let $0<\mu<1$ and assume that the conductivity a is uniformly elliptic in $D$ and belongs to $\mathcal{C}^{\mu}\left(\bar{D}_{l}\right), 1 \leqslant l \leqslant L$. Let $h \in \mathcal{C}^{0, \mu}\left(\bar{D}_{l}\right), 1 \leqslant l \leqslant L, f \in L^{\infty}(D)$ and let $u$ be a solution in $D$ to the equation

$$
-\operatorname{div}(a(x) \nabla u)=f+\operatorname{div}(h) .
$$

For any $\eta>0$, there exists a constant $C$ such that, for any

$$
0<\alpha^{\prime} \leqslant \min \left\{\mu, \frac{\alpha}{2(\alpha+1)}\right\}
$$


u satisfies

$$
\sum_{l=1}^{L}\|u\|_{\mathcal{C}^{1, \alpha^{\prime}}\left(\bar{D}_{l} \cap D_{\eta}\right)} \leqslant C\left(\|u\|_{L^{2}(D)}+\|f\|_{L^{\infty}(D)}+\sum_{l=1}^{L}\|h\|_{\mathcal{C}^{\alpha^{\prime}\left(\bar{D}_{l}\right)}}\right) .
$$

Here $C$ depends only on $\lambda, \Lambda, \mu, L, \alpha, \eta,\|a\|_{\mathcal{C}^{\alpha^{\prime}}\left(\bar{D}_{l}\right)}$ and on the $\mathcal{C}^{1, \alpha^{\prime}}$ norm of the $D_{l}$. In particular,

$$
\|\nabla u\|_{L^{\infty}\left(D_{\eta}\right)} \leqslant C\left(\|u\|_{L^{2}(D)}+\|f\|_{L^{\infty}(D)}+\sum_{l=1}^{L}\|h\|_{\mathcal{C}^{\alpha^{\prime}\left(\bar{D}_{l}\right)}}\right) .
$$

In the following, for each $r, 0<r<1$, and $x \in \mathbb{R}^{3}$, we set

$$
B(x, r)=\left\{y \in \mathbb{R}^{3} /|x-y|<r\right\} \quad \text { and } \quad B_{r}=B(0, r) .
$$

The constant $C$ in the above theorem may, however, grow with the number of inclusions. However, in the case of periodic media, uniform pointwise estimates on the gradients do hold as in theorem 3.1. This is established in the following result, due to $\mathrm{Li}$ and Nirenberg (see also the remark in $\S 5.3$ of [4]). Its proof relies on theorem 3.2 and on the three-step compactness method of [4].

TheOREM 3.3 (Li and Nirenberg [15, theorem 0.2]). Assume that

$$
a \in \mathcal{A}(\lambda, \Lambda, \mu, \alpha) \quad \text { and } \quad L_{\varepsilon} \in \mathcal{L}(\lambda, \Lambda, \mu, \alpha) .
$$

Let $u_{\varepsilon}$ be a solution to

$$
L_{\varepsilon} u_{\varepsilon}=0 \quad \text { in } B_{1} \text {. }
$$

Then

$$
\left\|\nabla u_{\varepsilon}\right\|_{L^{\infty}\left(B_{1 / 2}\right)} \leqslant C\left\|u_{\varepsilon}\right\|_{L^{2}\left(B_{1}\right)},
$$

where $C$ is independent of $\varepsilon$ (and thus is independent of the number of inclusions and of the distance between their boundaries).

We will need a slightly different version of theorem 3.3 for solutions of elliptic equations in divergence form, with a source term of the following particular form.

THEOREM 3.4 (interior gradient estimates). Assume that

$$
\mu \leqslant \frac{\alpha}{2(\alpha+1)} .
$$

Let $a \in \mathcal{A}(\lambda, \Lambda, \mu, \alpha), f \in L^{\infty}\left(B_{1}\right), h \in \mathcal{C}^{0, \mu}\left(B_{1}\right)^{3}$ and $L_{\varepsilon} \in \mathcal{L}(\lambda, \Lambda, \mu, \alpha)$. Let $b$ be a $Y$-periodic function such that $b$ has regularity $\mathcal{C}^{0, \mu}$ in $\bar{D}_{0}$ and in $\overline{Y \backslash D_{0}}$. Assume that $u_{\varepsilon}$ is a solution to

$$
L_{\varepsilon} u_{\varepsilon}=f+\varepsilon \operatorname{div}\left(b_{\varepsilon} h\right) \quad \text { in } B_{1} .
$$

Then

$$
\left\|u_{\varepsilon}\right\|_{\mathcal{C}^{0, \mu}\left(B_{1 / 2}\right)}+\left\|\nabla u_{\varepsilon}\right\|_{L^{\infty}\left(B_{1 / 2}\right)} \leqslant C\left(\left\|u_{\varepsilon}\right\|_{L^{2}\left(B_{1}\right)}+\|f\|_{L^{\infty}\left(B_{1}\right)}+\|h\|_{\mathcal{C}^{0, \mu}\left(B_{1}\right)}\right),
$$

where $C$ is independent of $\varepsilon$ (and thus of the number of inclusions and of the distances between their boundaries). 
On the basis of theorem 3.4, one can proceed as in [5], and generalize to composite media with sufficiently smooth inclusions, error estimates between the potential $u_{\varepsilon}$ and the homogenized potential $u_{0}$, and between the $\varepsilon$-periodic Green function and the Green function for the homogenized medium.

However, as we intend to apply such results to Neumann problems, we first state the following $L^{2}$ error estimate.

LEMMA 3.5. Assume that $\Omega$ is a smooth bounded domain in $\mathbb{R}^{3}$ and that $g \in$ $\mathcal{C}^{\infty}(\partial \Omega)$ such that $\int_{\partial \Omega} g=0$. Let $u_{\varepsilon}$ denote the solution to

$$
\left.\begin{array}{rl}
\operatorname{div}\left(a_{\varepsilon} \nabla u_{\varepsilon}\right)=0 & \text { in } \Omega, \\
a_{\varepsilon} \nabla u_{\varepsilon} \cdot \nu=g & \text { on } \partial \Omega,
\end{array}\right\}
$$

normalized with the condition that $\int_{\Omega} u_{\varepsilon}=0$. Let $u_{0}$ denote the solution to the corresponding homogenized problem

$$
\left.\begin{array}{rl}
\operatorname{div}\left(A \nabla u_{0}\right)=0 & \text { in } \Omega, \\
A \nabla u_{0} \cdot \nu=g & \text { on } \partial \Omega,
\end{array}\right\}
$$

also normalized by $\int_{\Omega} u_{0}=0$. Then the following estimate holds

$$
\left\|u_{\varepsilon}-u_{0}\right\|_{L^{2}(\Omega)} \leqslant C \varepsilon\left\|u_{0}\right\|_{H^{2}(\Omega)} .
$$

Estimates of this sort are well known for Dirichlet boundary conditions $[1,6,19]$. For Neumann boundary conditions, a similar error estimate was derived in two dimensions by Moskow and Vogelius [20] in the case of a convex polygon using harmonic conjugates. We show in $\S \mathrm{A} .2$ how this estimate generalizes to three dimensions.

We now state uniform error estimates between $u_{\varepsilon}$ and $u_{0}$.

TheOREm 3.6. Let $\omega \subset \subset \Omega$. Assume that $u_{\varepsilon}$ and $u_{0}$ solve $L_{\varepsilon} u_{\varepsilon}=0$ and $L_{0} u_{0}=0$ in $\Omega$. Assume also that

$$
\left\|u_{\varepsilon}-u_{0}\right\|_{L^{2}(\Omega)} \leqslant C \varepsilon^{\sigma},
$$

for some $0<\sigma \leqslant 1$. There then exists a constant $C$ that depends only on $\lambda, \Lambda, \mu$, $\alpha, \Omega$ and $\omega$ such that

$$
\begin{array}{r}
\left\|u_{\varepsilon}-u_{0}\right\|_{L^{\infty}(\omega)} \leqslant C \varepsilon^{\sigma}, \\
\left\|\nabla u_{\varepsilon}-\left(I+\nabla_{y} \chi(\cdot / \varepsilon)\right) \nabla u_{0}\right\|_{L^{\infty}(\omega)} \leqslant C \varepsilon^{\sigma} .
\end{array}
$$

We remark that, by lemma 3.5, this theorem applies to solutions of (3.16), (3.17).

Let $G_{\varepsilon}$ and $G_{0}$ denote the respective Green functions, vanishing on $\partial \Omega$, of the operators $L_{\varepsilon}$ and $L_{0}$. From theorem 3.6, we derive an estimate on the convergence rate of $G_{\varepsilon}$ to $G_{0}$. This result is applied, in $\S 4$, when the source is far from the defect. For this reason, we consider below the case $G_{\varepsilon}(x, y)$ when $x \in \omega \subset \subset \Omega$ and $y \in \Omega \backslash \omega$ with $\operatorname{dist}(y, \omega)>0$. In [5], when the coefficients have Hölder regularity, similar estimates are established that are valid on the whole of $\Omega$ (away from the source). Their derivation requires uniform boundary estimates on $L_{\varepsilon}$-harmonic functions. It would be interesting to study whether such estimates also hold in our context. 
TheOREM 3.7. Assume that $\omega \subset \subset \Omega$ is a smooth domain. Let $G_{\varepsilon}$ and $G_{0}$ be the Green functions, vanishing on $\partial \Omega$, for the operators $L_{\varepsilon}$ and $L_{0}$ (see $\left.\S 2\right)$. There exists a positive constant $C$, independent of $\varepsilon$, such that, for $y \in \Omega \backslash \omega$ with $\operatorname{dist}(y, \omega)>0$,

$$
\begin{aligned}
\left\|G_{\varepsilon}(\cdot, y)-G_{0}(\cdot, y)\right\|_{L^{\infty}(\omega)} & \leqslant C \varepsilon^{1 / 4}, \\
\left\|\nabla_{x} G_{\varepsilon}(\cdot, y)-\left(I+\nabla_{y} \chi(\cdot / \varepsilon)\right) \nabla_{x} G_{0}(\cdot, y)\right\|_{L^{\infty}(\omega)} & \leqslant C \varepsilon^{1 / 4} .
\end{aligned}
$$

\section{Main result: asymptotics of the perturbed potential}

Let $Y$ denotes the unit cell $(0,1)^{3}$ in $\mathbb{R}^{3}$. We assume that $Y$ contains an inclusion $D_{0}$, the boundary of which has regularity $\mathcal{C}^{1, \alpha}$ for some $0<\alpha<1$. We also assume that

$$
\operatorname{dist}\left(D_{0}, \partial Y\right) \geqslant d_{0}>0 .
$$

Let $a$ be a measurable $Y$-periodic function equal to a constant $k$ in $D_{0}, 0<k<\infty$, $k \neq 1$, and equal to 1 in $Y \backslash \bar{D}_{0}$.

As in $\S 3$, we consider a bounded domain $\Omega \subset \mathbb{R}^{3}$ formed by the union of cells, translated and rescaled by $\varepsilon$ from the elementary cell $Y$. The conductivity in $\Omega$ is denoted by $a_{\varepsilon}(x)=a(x / \varepsilon)$. We consider the elliptic operator $L_{\varepsilon}=-\operatorname{div}\left(a_{\varepsilon}(x) \nabla \cdot\right)$. We call the solution $u_{\varepsilon}$ to

$$
\left.\begin{array}{c}
L_{\varepsilon} u_{\varepsilon}=0 \quad \text { in } \Omega, \\
\left.a_{\varepsilon} \frac{\partial u_{\varepsilon}}{\partial \nu}\right|_{\partial \Omega}=g, \\
\int_{\partial \Omega} g \mathrm{~d} \sigma_{x}=\int_{\Omega} u_{\varepsilon} \mathrm{d} x=0
\end{array}\right\}
$$

background electrostatic potential in $\Omega$. We study the influence of a particular perturbation of such a medium which consists in misplacing one inclusion. More precisely, let $p \in \mathbb{Z}^{3}$ such that $Y_{\varepsilon}^{p}:=\varepsilon(p+Y) \subset \Omega$, and so that $\operatorname{dist}\left(Y_{\varepsilon}^{p}, \partial \Omega\right) \gg \varepsilon$. If the medium were completely periodic, the inclusion contained in the cell $Y_{\varepsilon}^{p}$ would occupy the subset $\omega_{\varepsilon, 1}:=\varepsilon\left(p+D_{0}\right)$. Instead, the inclusion lies in a subset $\omega_{\varepsilon, 2}=\varepsilon\left(p+\delta+D_{0}\right)$, for some $0<|\delta|<1$. For simplicity, we assume that $\omega_{\varepsilon, 2}$ does not intersect any of the remaining inclusions. Let $\omega_{\varepsilon}$ denote the symmetric difference of the sets $\omega_{\varepsilon, 1}$ and $\omega_{\varepsilon, 2}$.

The conductivity $a_{\varepsilon, d}$ of the perturbed medium is thus given by

$$
a_{\varepsilon, d}= \begin{cases}a_{\varepsilon} & \text { in } \Omega \backslash \bar{\omega}_{\varepsilon}, \\ 1 & \text { in } \omega_{\varepsilon, 1} \backslash \omega_{\varepsilon, 2}, \\ k & \text { in } \omega_{\varepsilon, 2} \backslash \omega_{\varepsilon, 1} .\end{cases}
$$

The associated potential $u_{\varepsilon, d}$ solves

$$
\left.\begin{array}{c}
\operatorname{div}\left(a_{\varepsilon, d}(x) \nabla u_{\varepsilon, d}\right)=0 \quad \text { in } \Omega, \\
\left.a_{\varepsilon, d} \frac{\partial u_{\varepsilon, d}}{\partial \nu}\right|_{\partial \Omega}=g, \\
\int_{\partial \Omega} g \mathrm{~d} \sigma_{x}=\int_{\Omega} u_{\varepsilon, d} \mathrm{~d} x=0 .
\end{array}\right\}
$$


Without loss of generality, we assume that $x_{0}=0$ belongs to the convex hull of $\omega_{\varepsilon}$. Let

$$
\tilde{\Omega}_{\varepsilon}=\left\{\frac{x}{\varepsilon}, x \in \Omega\right\}, \quad \tilde{\omega}=\left\{\frac{x}{\varepsilon}, x \in \omega_{\varepsilon}\right\}
$$

(note that $\tilde{\omega}$ is independent of $\varepsilon$ ), and define the function $a_{d}$ in $\mathbb{R}^{3}$ by $a_{d}(y)=$ $a_{\varepsilon, d}(\varepsilon y)$. Throughout this section, we denote by $a^{+}(x)$ and $a^{-}(x)$ the outward and inward limits, respectively, of the discontinuous function $a$ through an interface.

Let $G_{\varepsilon}$ be the Green function associated with the operator $L_{\varepsilon}$, the solution to

$$
-\operatorname{div}\left(a_{\varepsilon} \nabla G_{\varepsilon}(x, z)\right)=\delta_{z} \quad \text { in } \Omega,
$$

vanishing on $\partial \Omega$ (see $\S 2$ ), and $G_{0}$ be the Green function of the homogenized operator $L_{0}$, defined by (3.8).

We now state the main result of this paper: an asymptotic expansion for $u_{\varepsilon, d}-u_{\varepsilon}$. This expansion has the same structure as that derived in the case of a homogeneous background medium [10], although it involves the homogenized potential $u_{0}$ and the homogenized Green function $G_{0}$. As mentioned in $\S 1$, the presence of the Green function (and its singularity) should make this expansion useful for numerical detection purposes.

We note that our analysis extends easily to the case of several misplaced inclusions (or to the case of $O(\varepsilon)$ defects with different constant conductivities), provided that they are at distances greater than $O(\varepsilon)$ apart.

THEOREM 4.1. Assume that $\Omega$ and the Neumann data $g$ are sufficiently regular that the homogenized potential $u_{0}$ is smooth inside $\Omega$. For any $z \in \Omega$ at a distance $d_{0}>0$ away from $\omega_{\varepsilon}$, we have

$$
\begin{aligned}
u_{\varepsilon, d}(z)-u_{\varepsilon}(z)+\int_{\partial \Omega}\left(u_{\varepsilon, d}(x)\right. & \left.-u_{\varepsilon}(x)\right) \frac{\partial G_{\varepsilon}}{\partial \nu_{x}} \mathrm{~d} \sigma_{x} \\
& =-\varepsilon^{3} \nabla_{x} G_{0}\left(x_{0}, z\right) \cdot M \nabla_{x} u_{0}\left(x_{0}\right)+O\left(\varepsilon^{3+(1 / 4)}\right) .
\end{aligned}
$$

The term $O\left(\varepsilon^{3+(1 / 4)}\right)$ is uniformly bounded by $C \varepsilon^{3+(1 / 4)}$, where the constant $C$ depends on $d_{0}, k$ and $\alpha$. The polarization tensor $M$ is given by

$$
M_{i j}=\int_{\partial \tilde{\omega}}\left(\frac{a^{-}}{a_{d}^{-}}-1\right)\left(y_{i}+\chi^{i}(y)\right)\left(a^{+}(y) \frac{\partial \varphi_{j, d}^{+}}{\partial \nu_{y}}+a^{-}(y)\left(\nu_{j}+\frac{\partial \chi^{j}(y)}{\partial \nu_{y}}\right)\right) \mathrm{d} \sigma_{y}
$$

for $1 \leqslant i, j \leqslant 3$, where the cell function $\chi=\left(\chi^{i}\right)_{1 \leqslant i \leqslant 3}$ is defined by (3.4) and where the auxiliary functions $\varphi_{j, d}$ are defined by (4.14) below.

To prove the theorem, we first establish three lemmas. We introduce two auxiliary functions $v_{\varepsilon, d}$ and $v_{d}$, respective solutions to

$$
\begin{aligned}
& \operatorname{div}\left(a(y) \nabla_{y} v_{\varepsilon, d}\right)=0 \quad \text { in } \tilde{\Omega}_{\varepsilon} \backslash \overline{\tilde{\omega}}, \quad \Delta v_{\varepsilon, d}=0 \quad \text { in } \tilde{\omega}, \\
& v_{\varepsilon, d}^{+}-v_{\varepsilon, d}^{-}=0 \quad \text { on } \partial \tilde{\omega}, \\
& a^{+} \frac{\partial v_{\varepsilon, d}^{+}}{\partial \nu}-a_{d}^{-} \frac{\partial v_{\varepsilon, d}^{-}}{\partial \nu}=-\left(a^{-}-a_{d}^{-}\right)\left(I+\nabla_{y} \chi^{-}\right) \nabla_{x} u_{0}(0) \cdot \nu_{y} \quad \text { on } \partial \tilde{\omega}, \\
& a_{d} \frac{\partial v_{\varepsilon, d}}{\partial \nu_{y}}=0 \quad \text { on } \partial \tilde{\Omega}_{\varepsilon}, \quad \int_{\tilde{\Omega}_{\varepsilon}} v_{\varepsilon, d}=0,
\end{aligned}
$$


and

$$
\left.\begin{array}{c}
\operatorname{div}_{y}\left(a_{d}(y) \nabla_{y} v_{d}\right)=\mu \quad \text { in } \mathbb{R}^{3}, \\
v_{d}(y) \rightarrow 0 \quad \text { when }|y| \rightarrow \infty,
\end{array}\right\}
$$

with $\mu=\operatorname{div}_{y}\left(\left(a_{d}-a\right)\left(I+\nabla_{y} \chi\right) \nabla_{x} u_{0}(0)\right)$.

Lemma 4.2. The function $v_{d}$, the solution of (4.7), decays at infinity as

$$
\left.\begin{array}{rl}
v_{d}(y) & =O\left(|y|^{-1}\right), \\
\nabla_{y} v_{d}(y) & =O\left(|y|^{-2}\right) .
\end{array}\right\}
$$

Proof. Since the support of $\mu$ is included in $\tilde{\omega}$, the function $v_{d}$ can be represented in terms of the Green function $G$ associated with $L=-\operatorname{div}_{y}\left(a_{d}(y) \nabla_{y} \cdot\right)$ in $\mathbb{R}^{3}$

$$
v_{d}(y)=\int_{\mathbb{R}^{3}} G(y, z) \mathrm{d} \mu(z)=\int_{\tilde{\omega}} G(y, z) \mathrm{d} \mu(z)
$$

(see [18, theorem 6.1$]$, where it is shown that the integral on the right-hand side of (4.9) exists a.e. as a consequence of Fubini's theorem).

It follows from $(2.8)$ that

$$
v_{d}(y)=O\left(|y|^{-1}\right) \quad \text { as }|y| \rightarrow \infty .
$$

Fix $z \in \mathbb{R}^{3}$ and $R>4 \operatorname{diam}(\tilde{\omega})$. As a function of $y, G(y, z)$ satisfies

$$
\operatorname{div}_{y}\left(a_{d}(y) \nabla_{y} G(y, z)\right)=0 \quad \text { in } B(z, 4 R) \backslash B\left(z, \frac{1}{4} R\right) .
$$

Thus, the rescaled function $g(x, z / R)=G(R x, z)$ solves

$$
\operatorname{div}_{x}\left(a_{d}(R x) \nabla_{x} g\left(x, \frac{z}{R}\right)\right)=0
$$

in the set $B(z / R, 4) \backslash B\left(z / R, \frac{1}{4}\right)$. Applying theorem 3.4 and (2.8), we obtain

$$
\begin{aligned}
\left\|\nabla_{y} G(y, z)\right\|_{L^{\infty}(B(z, 2 R) \backslash B(z, R / 2))} & \leqslant R^{-1}\left\|\nabla_{x} g\left(\frac{y}{R}, \frac{z}{R}\right)\right\|_{L^{\infty}(B(z / R, 2) \backslash B(z / R, 1 / 2))} \\
& \leqslant C R^{-1}\left\|g\left(\cdot, \frac{z}{R}\right)\right\|_{L^{\infty}(B(z / R, 4) \backslash B(z / R, 1 / 4))} \\
& \leqslant C R^{-1}\|G(\cdot, z)\|_{L^{\infty}(B(z, 4 R) \backslash B(z, R / 4))} \\
& \leqslant C R^{-1} \sup \left\{|y-z|^{-1}, y \in B(z, 4 R) \backslash B\left(z, \frac{1}{4} R\right)\right\} .
\end{aligned}
$$

It follows that

$$
\left|\nabla_{y} G(y, z)\right|=O\left(|y-z|^{-2}\right) \quad \text { as }|y-z| \rightarrow \infty .
$$

Noting that $G(\cdot, z)$ is $\mathcal{C}^{1, \mu}$ away from $y=z$ as a consequence of theorem 3.2, we can differentiate (4.9) with respect to $y$, and we conclude from (4.10) that

$$
\left|\nabla_{y} v_{d}(y)\right|=O\left(|y|^{2}\right)
$$

which proves the lemma. 
LEMma 4.3. There exists a constant $C>0$, independent of $\varepsilon$, such that

$$
\left\|\nabla_{y}\left(u_{\varepsilon, d}(\varepsilon \cdot)-u_{\varepsilon}(\varepsilon \cdot)-\varepsilon v_{\varepsilon, d}\right)\right\|_{L^{2}\left(\tilde{\Omega}_{\varepsilon}\right)} \leqslant C \varepsilon^{2} .
$$

Proof. Let $z_{\varepsilon, d}(y)=u_{\varepsilon, d}(\varepsilon y)-u_{\varepsilon}(\varepsilon y)-\varepsilon v_{\varepsilon, d}(y)$. According to equations (4.1), (4.2), $z_{\varepsilon, d}$ satisfies the following:

$$
\begin{aligned}
& \operatorname{div}\left(a(y) \nabla_{y} z_{\varepsilon, d}\right)=0 \quad \text { in } \tilde{\Omega}_{\varepsilon} \backslash \overline{\tilde{\omega}} \text { and in } \tilde{\omega}, \\
& z_{\varepsilon, d}^{+}-z_{\varepsilon, d}^{-}=0 \quad \text { on } \partial \tilde{\omega} \\
& a_{d}^{+} \frac{\partial z_{\varepsilon, d}^{+}}{\partial \nu}-a_{d}^{-} \frac{\partial z_{\varepsilon, d}^{-}}{\partial \nu}=-\varepsilon\left(a^{-}-a_{d}^{-}\right)\left(\nabla_{x} u_{\varepsilon}^{-}(\varepsilon y)-\left(I+\nabla_{y} \chi^{-}(y)\right) \nabla_{x} u_{0}(0)\right) \cdot \nu_{y}, \\
& a_{d} \frac{\partial z_{\varepsilon, d}}{\partial \nu_{y}}=0 \quad \text { on } \partial \tilde{\Omega}_{\varepsilon}
\end{aligned}
$$

Thus, integrating by parts yields

$$
\begin{aligned}
\int_{\tilde{\Omega}_{\varepsilon}} a_{d}(y) & \nabla_{y} z_{\varepsilon, d} \nabla_{y} z_{\varepsilon, d} \mathrm{~d} y \\
& =\int_{\tilde{\Omega}_{\varepsilon} \backslash \tilde{\omega}} a_{d}(y) \nabla_{y} z_{\varepsilon, d} \nabla_{y} z_{\varepsilon, d} \mathrm{~d} y+\int_{\tilde{\omega}} a_{d}(y) \nabla_{y} z_{\varepsilon, d} \nabla_{y} z_{\varepsilon, d} \mathrm{~d} y \\
& =-\int_{\partial \tilde{\omega}} a_{d}^{+} \frac{\partial z_{\varepsilon, d}^{+}}{\partial \nu_{y}} z_{\varepsilon, d}+\int_{\partial \tilde{\omega}} a_{d}^{-} \frac{\partial z_{\varepsilon, d}^{-}}{\partial \nu_{y}} z_{\varepsilon, d} \\
& =-\varepsilon \int_{\partial \tilde{\omega}}\left(a^{-}(y)-a_{d}^{-}(y)\right)\left(\nabla_{x} u_{\varepsilon}^{-}(\varepsilon y)-\left(I+\nabla_{y} \chi^{-}(y)\right) \nabla_{x} u_{0}(0)\right) \cdot \nu_{y} z_{\varepsilon, d} \\
& =\varepsilon \int_{\tilde{\omega}}\left(a(y)-a_{d}(y)\right)\left(\nabla_{x} u_{\varepsilon}(\varepsilon y)-\left(I+\nabla_{y} \chi(y)\right) \nabla_{x} u_{0}(0)\right) \cdot \nabla_{y} z_{\varepsilon, d} \\
& \leqslant c \varepsilon\left\|\nabla_{x} u_{\varepsilon}(\varepsilon \cdot)-\left(I+\nabla_{y} \chi\right) \nabla_{x} u_{0}(0)\right\|_{L^{2}(\tilde{\omega})}\left\|\nabla_{y} z_{\varepsilon, d}\right\|_{L^{2}(\tilde{\omega})} .
\end{aligned}
$$

Lemma 3.5, theorem 3.6 and the smoothness of the homogenized potential $u_{0}$ show that

$$
\begin{aligned}
& \left\|\nabla_{x} u_{\varepsilon}-\left(I+\nabla_{y} \chi(\cdot / \varepsilon)\right) \nabla_{x} u_{0}(0)\right\|_{L^{\infty}\left(\omega_{\varepsilon}\right)} \\
& \leqslant\left\|\nabla_{x} u_{\varepsilon}-\left(I+\nabla_{y} \chi(\cdot / \varepsilon)\right) \nabla_{x} u_{0}(\cdot)\right\|_{L^{\infty}\left(\omega_{\varepsilon}\right)} \\
& \quad+\left\|\left(I+\nabla_{y} \chi(\cdot / \varepsilon)\right)\left(\nabla_{x} u_{0}(\cdot)-\nabla_{x} u_{0}(0)\right)\right\|_{L^{\infty}\left(\omega_{\varepsilon}\right)} \\
& \quad \leqslant C \varepsilon .
\end{aligned}
$$

Since $a_{\varepsilon}$ is bounded, we conclude that

$$
\left\|\nabla_{y} z_{\varepsilon, d}\right\|_{L^{2}\left(\tilde{\Omega}_{\varepsilon}\right)}^{2} \leqslant c \varepsilon^{2}\left\|\nabla_{y} z_{\varepsilon, d}\right\|_{L^{2}(\tilde{\omega})} \leqslant c \varepsilon^{2}\left\|\nabla_{y} z_{\varepsilon, d}\right\|_{L^{2}\left(\tilde{\Omega}_{\varepsilon}\right)} .
$$

Lemma 4.4. There exists a constant $C$, independent of $\varepsilon$, such that

$$
\left\|\nabla_{y}\left(u_{\varepsilon, d}(\varepsilon \cdot)-u_{\varepsilon}(\varepsilon \cdot)-\varepsilon v_{d}\right)\right\|_{L^{2}\left(\tilde{\Omega}_{\varepsilon}\right)} \leqslant C \varepsilon^{3 / 2} .
$$


Proof. Lemma 4.3 shows that it is sufficient to prove that

$$
\left\|\nabla_{y}\left(v_{\varepsilon, d}-v_{d}\right)\right\|_{L^{2}\left(\tilde{\Omega}_{\varepsilon}\right)} \leqslant c \varepsilon^{1 / 2} .
$$

To this end, let $\phi_{\varepsilon, d}=v_{\varepsilon, d}(y)-v_{d}(y)-c_{\varepsilon}$, where $c_{\varepsilon}$ is chosen so that

$$
\int_{\partial \tilde{\Omega}_{\varepsilon}} \phi_{\varepsilon, d}=0
$$

In view of (4.6) and (4.7), $\phi_{\varepsilon, d}$ solves

$$
\begin{aligned}
\operatorname{div}\left(a_{d}(y) \nabla \phi_{\varepsilon, d}\right) & =0 \quad \text { in } \tilde{\Omega}_{\varepsilon} \\
a_{d}(y) \frac{\partial \phi_{\varepsilon, d}}{\partial \nu_{y}} & =-a_{d}(y) \frac{\partial v_{d}}{\partial \nu_{y}} \quad \text { on } \partial \tilde{\Omega}_{\varepsilon}, \\
\int_{\partial \tilde{\Omega}_{\varepsilon}} \phi_{\varepsilon, d} & =0 .
\end{aligned}
$$

By integrating by parts and changing variables back to the fixed domain $\Omega$, we see that

$$
\begin{aligned}
\int_{\tilde{\Omega}_{\varepsilon}} a_{d}(y) \nabla_{y} \phi_{\varepsilon, d} \nabla_{y} \phi_{\varepsilon, d} \mathrm{~d} y & =\int_{\partial \tilde{\Omega}_{\varepsilon}}-a_{d} \frac{\partial v_{d}}{\partial \nu_{y}} \phi_{\varepsilon, d} \mathrm{~d} \sigma_{y} \\
& =\varepsilon^{-2} \int_{\partial \Omega}-a_{d} \frac{\partial v_{d}}{\partial \nu_{y}}\left(\frac{x}{\varepsilon}\right) \phi_{\varepsilon, d}\left(\frac{x}{\varepsilon}\right) \mathrm{d} \sigma_{x} \\
& \leqslant c \varepsilon^{-2}\left\|\frac{\partial v_{d}}{\partial \nu_{y}}(\cdot / \varepsilon)\right\|_{L^{\infty}(\partial \Omega)}\left\|\phi_{\varepsilon, d}(\cdot / \varepsilon)\right\|_{L^{2}(\partial \Omega)} .
\end{aligned}
$$

The trace theorem and the Poincaré-Wirtinger inequality imply that

$$
\left\|\phi_{\varepsilon, d}(\cdot / \varepsilon)\right\|_{L^{2}(\partial \Omega)} \leqslant C\left\|\phi_{\varepsilon, d}(\cdot / \varepsilon)\right\|_{W^{1,2}(\Omega)} \leqslant C \varepsilon^{1 / 2}\left\|\nabla_{y} \phi_{\varepsilon, d}\left(\frac{x}{\varepsilon}\right)\right\|_{L^{2}(\tilde{\Omega})} .
$$

Since $v_{d}$ decays at infinity (see $(4.8)$ ), we have

$$
\left\|\frac{\partial v_{d}}{\partial \nu_{y}}(\cdot / \varepsilon)\right\|_{L^{\infty}(\partial \Omega)}=O\left(\varepsilon^{2}\right)
$$

and therefore

$$
\begin{aligned}
\left\|\nabla_{y} \phi_{\varepsilon, d}\left(\frac{x}{\varepsilon}\right)\right\|_{L^{2}(\tilde{\Omega})}^{2} & \leqslant C \int_{\tilde{\Omega}} a_{\varepsilon}(\varepsilon y) \nabla_{y} \phi_{\varepsilon, d} \nabla_{y} \phi_{\varepsilon, d} \mathrm{~d} y \\
& \leqslant C \varepsilon^{1 / 2}\left\|\nabla_{y} \phi_{\varepsilon, d}\right\|_{L^{2}(\tilde{\Omega})}
\end{aligned}
$$

and the lemma is proved.

Proof of theorem 4.1. Let $u_{\varepsilon, d}$ and $u_{\varepsilon}$ be the electrostatic-potential solutions to (4.2) and (4.1), respectively. Let $z$ be a point in $\Omega$, at a distance $d>0$ away from 
$\omega_{\varepsilon}$. We apply the Green formula in $\Omega$ to get

$$
\begin{aligned}
u_{\varepsilon, d}(z)= & -\int_{\Omega} u_{\varepsilon, d} \operatorname{div}\left(a_{\varepsilon}(x) \nabla G_{\varepsilon}(x, z)\right) \mathrm{d} x \\
= & \int_{\Omega \backslash \omega_{\varepsilon}} a_{\varepsilon}(x) \nabla_{x} u_{\varepsilon, d} \nabla_{x} G_{\varepsilon} \mathrm{d} x+\int_{\omega_{\varepsilon}} a_{\varepsilon}(x) \nabla_{x} u_{\varepsilon, d} \nabla_{x} G_{\varepsilon} \mathrm{d} x \\
& -\int_{\partial \Omega} u_{\varepsilon, d} a_{\varepsilon}(x) \frac{\partial G_{\varepsilon}}{\partial \nu_{x}} \mathrm{~d} \sigma_{x} \\
= & \int_{\partial \Omega} g G_{\varepsilon} \mathrm{d} \sigma_{x}-\int_{\partial \Omega} u_{\varepsilon, d} a_{\varepsilon} \frac{\partial G_{\varepsilon}}{\partial \nu_{x}} \mathrm{~d} \sigma_{x} \\
& \quad-\int_{\partial \omega_{\varepsilon}}\left(a_{\varepsilon}^{+} \frac{\partial u_{\varepsilon, d}^{+}}{\partial \nu_{x}}-a_{\varepsilon}^{-} \frac{\partial u_{\varepsilon, d}^{-}}{\partial \nu_{x}}\right) G_{\varepsilon} \mathrm{d} \sigma_{x}
\end{aligned}
$$

and

$$
u_{\varepsilon}(z)=\int_{\partial \Omega} g G_{\varepsilon} \mathrm{d} \sigma_{x}-\int_{\partial \Omega} u_{\varepsilon} a_{\varepsilon} \frac{\partial G_{\varepsilon}}{\partial \nu_{x}} \mathrm{~d} \sigma_{x} .
$$

Using the continuity of $u_{\varepsilon, d}$ and the jump conditions satisfied by its normal derivative across $\partial \omega_{\varepsilon}$, the difference between these two equations yields

$$
\begin{aligned}
u_{\varepsilon, d}(z)- & u_{\varepsilon}(z)-\int_{\partial \Omega}\left(u_{\varepsilon}-u_{\varepsilon, d}\right) a_{\varepsilon} \frac{\partial G_{\varepsilon}}{\partial \nu_{x}} \mathrm{~d} \sigma_{x} \\
& =\int_{\partial \omega_{\varepsilon}}\left(a_{\varepsilon}^{-}-a_{\varepsilon, d}^{-}\right) \frac{\partial u_{\varepsilon, d}^{-}}{\partial \nu_{x}} G_{\varepsilon} \mathrm{d} \sigma_{x} \\
& =\int_{\partial \omega_{\varepsilon}}\left(a_{\varepsilon}^{-}-a_{\varepsilon, d}^{-}\right)\left(\frac{\partial u_{\varepsilon}^{-}}{\partial \nu_{x}}+\frac{\partial v_{d}^{-}}{\partial \nu_{y}}\right) G_{\varepsilon} \mathrm{d} \sigma_{x}+\int_{\partial \omega_{\varepsilon}}\left(a_{\varepsilon}^{-}-a_{\varepsilon, d}^{-}\right) \frac{\partial r_{\varepsilon, d}^{-}}{\partial \nu_{x}} G_{\varepsilon} \mathrm{d} \sigma_{x} \\
& =I_{1}+I_{2},
\end{aligned}
$$

where $r_{\varepsilon, d}(x)=u_{\varepsilon, d}(x)-u_{\varepsilon}(x)-\varepsilon v_{d}(x / \varepsilon)$.

Combining the $W^{1, \infty}$-error estimate (3.21) for the Green function $G_{\varepsilon}$ and the fact that $\nabla_{x} u_{\varepsilon}$ is bounded on every compact subset of $\Omega$ that contains $\omega_{\varepsilon}$ (theorem 3.4), we show that

$$
\begin{aligned}
& \int_{\partial \omega_{\varepsilon}}\left(a_{\varepsilon}^{-}\right.\left.-a_{\varepsilon, d}^{-}\right)\left(\frac{\partial u_{\varepsilon}^{-}}{\partial \nu_{x}}+\frac{\partial v_{d}^{-}}{\partial \nu_{y}}\right)\left(G_{\varepsilon}(x, z)-G_{0}(x, z)-\varepsilon \nabla G_{0}(x, z) \chi\left(\frac{x}{\varepsilon}\right)\right) \mathrm{d} \sigma_{x} \\
& \leqslant C\left\|\nabla_{x} u_{\varepsilon}+\nabla_{y} v_{d}\right\|_{L^{2}\left(\omega_{\varepsilon}\right)}\left\|\nabla_{x} G_{\varepsilon}-\left(I+\nabla_{y} \chi\left(\frac{x}{\varepsilon}\right)\right) \nabla G_{0}(x)\right\|_{L^{2}\left(\omega_{\varepsilon}\right)} \\
& \leqslant C \varepsilon^{3 / 2}\left(\left\|\nabla_{x} u_{\varepsilon}\right\|_{L^{\infty}\left(\omega_{\varepsilon}\right)}+\left\|\nabla_{y} v_{d}\right\|_{L^{2}(\tilde{\omega})}\right) \\
& \quad \times \varepsilon^{3 / 2}\left\|\nabla_{x} G_{\varepsilon}-\left(I+\nabla_{y} \chi\left(\frac{x}{\varepsilon}\right)\right) \nabla G_{0}(x)\right\|_{L^{\infty}\left(\omega_{\varepsilon}\right)} \\
& \leqslant C \varepsilon^{3+(1 / 4)} .
\end{aligned}
$$


Hence,

$$
\begin{aligned}
I_{1}= & \int_{\partial \omega_{\varepsilon}}\left(a_{\varepsilon}^{-}(x)-a_{\varepsilon, d}^{-}(x)\right)\left(\frac{\partial u_{\varepsilon}^{-}}{\partial \nu_{x}}+\frac{\partial v_{d}^{-}}{\partial \nu_{y}}\right) G_{\varepsilon} \mathrm{d} \sigma_{x} \\
= & \varepsilon^{2} \int_{\partial \tilde{\omega}}\left(a^{-}(y)-a_{d}^{-}(y)\right)\left(\frac{\partial u_{\varepsilon}^{-}}{\partial \nu_{x}}+\frac{\partial v_{d}^{-}}{\partial \nu_{y}}\right)\left(G_{0}(\varepsilon y, z)+\varepsilon \chi(y) \cdot \nabla_{x} G_{0}(\varepsilon y, z)\right) \mathrm{d} \sigma_{y} \\
& \quad+O\left(\varepsilon^{3+(1 / 4)}\right) .
\end{aligned}
$$

Thus, by a Taylor expansion of $G_{\varepsilon}$ about the origin,

$$
\begin{aligned}
I_{1}=\varepsilon^{2} & G_{0}(0, z) \int_{\partial \tilde{\omega}}\left(a^{-}-a_{d}^{-}\right)\left(\frac{\partial u_{\varepsilon}^{-}}{\partial \nu_{x}}+\frac{\partial v_{d}^{-}}{\partial \nu_{y}}\right) \mathrm{d} \sigma_{y} \\
& +\varepsilon^{3} \int_{\partial \tilde{\omega}}\left(a^{-}-a_{d}^{-}\right)\left(\frac{\partial u_{\varepsilon}^{-}}{\partial \nu_{x}}+\frac{\partial v_{d}^{-}}{\partial \nu_{y}}\right)\left(\nabla_{x} G_{0}(0, z) \cdot(y+\chi(y))\right) \mathrm{d} \sigma_{y} \\
& +O\left(\varepsilon^{3+(1 / 4)}\right) .
\end{aligned}
$$

Since $u_{\varepsilon, d}$ and $v_{d}$ are harmonic in $\tilde{\omega}$, the first term in the right-hand side of the above expression vanishes and $I_{1}$ reduces to

$$
I_{1}=\varepsilon^{3} \nabla_{x} G_{0}(0, z) \cdot \int_{\partial \tilde{\omega}}\left(a^{-}-a_{d}^{-}\right)\left(\frac{\partial u_{\varepsilon}^{-}}{\partial \nu_{x}}+\frac{\partial v_{d}^{-}}{\partial \nu_{y}}\right)(y+\chi(y)) \mathrm{d} \sigma_{y}+O\left(\varepsilon^{3+(1 / 4)}\right) .
$$

Invoking theorem 3.6 in a fixed subset $\omega \subset \subset \Omega$ that contains $\omega_{\varepsilon}$, we see that

$$
\left\|\nabla_{x} u_{\varepsilon}(\varepsilon y)-\left(I+\nabla_{y} \chi(y)\right) \nabla_{x} u_{0}(\varepsilon y)\right\|_{L^{\infty}(\tilde{\omega})} \leqslant C \varepsilon,
$$

for some constant $C$ independent of $\varepsilon$, and thus

$$
\begin{aligned}
\nabla_{x} G_{0}(0, z) & \cdot \int_{\partial \tilde{\omega}}\left(a^{-}-a_{d}^{-}\right) \frac{\partial u_{\varepsilon}^{-}}{\partial \nu_{x}}(y+\chi(y)) \mathrm{d} \sigma_{y} \\
& =\int_{\tilde{\omega}}\left(a^{-}-a_{d}^{-}\right) \nabla_{x} u_{\varepsilon} \cdot(I+\nabla \chi(y)) \nabla_{x} G_{0}(0, z) \mathrm{d} y \\
& =\int_{\tilde{\omega}}\left(a^{-}-a_{d}^{-}\right)(I+\nabla \chi(y)) \nabla_{x} u_{0}(\varepsilon y) \cdot(I+\nabla \chi) \nabla_{x} G_{0}(0, z) \mathrm{d} y+O(\varepsilon) \\
& =\nabla_{x} G_{0}(0, z) \int_{\partial \tilde{\omega}}\left(a^{-}-a_{d}^{-}\right)(I+\nabla \chi(y)) \nabla u_{0}(0) \cdot \nu(y+\chi(y)) \mathrm{d} \sigma_{y}+O(\varepsilon) .
\end{aligned}
$$

Thus, up to $O\left(\varepsilon^{3+(1 / 4)}\right)$, the term

$$
I_{1}=\varepsilon^{3} \nabla_{x} G_{0}(0, z) \cdot \int_{\partial \tilde{\omega}}\left(a^{-}-a_{d}^{-}\right)\left((I+\nabla \chi(y)) \nabla u_{0}(0) \cdot \nu+\frac{\partial v_{d}^{-}}{\partial \nu_{y}}\right)(y+\chi(y)) \mathrm{d} \sigma_{y} .
$$

Turning to $I_{2}$, integration by parts and the change of variables $y=x / \varepsilon$ give

$$
I_{2}=\int_{\partial \omega_{\varepsilon}}\left(a_{\varepsilon}^{-}-a_{\varepsilon, d}^{-}\right) \frac{\partial r_{\varepsilon, d}^{-}}{\partial \nu_{x}} G_{\varepsilon} \mathrm{d} \sigma_{x}=\varepsilon^{2} \int_{\tilde{\omega}}\left(a-a_{d}\right) \nabla_{y} r_{\varepsilon, d} \nabla G_{\varepsilon} \mathrm{d} y
$$

Lemma 4.4 shows that $\left\|\nabla_{y} r_{\varepsilon, d}(\varepsilon y)\right\|_{L^{2}\left(\tilde{\Omega}_{\varepsilon}\right)} \leqslant c \varepsilon^{3 / 2}$. Moreover, theorem 3.4 implies that $\nabla_{x} G_{\varepsilon}(x, z)$ is uniformly bounded in $\omega_{\varepsilon}$. Consequently,

$$
I_{2}=O\left(\varepsilon^{7 / 2}\right) .
$$


Thus, (4.13) yields

$$
\begin{aligned}
& u_{\varepsilon, d}(z)- u_{\varepsilon}(z)-\int_{\partial \Omega}\left(u_{\varepsilon}-u_{\varepsilon, d}\right) a_{\varepsilon} \frac{\partial G_{\varepsilon}}{\partial \nu_{x}} \mathrm{~d} \sigma_{x} \\
&=\varepsilon^{3} \nabla_{x} G_{0}(0, z) \cdot \int_{\partial \tilde{\omega}}\left(a^{-}-a_{d}^{-}\right)\left((I+\nabla \chi(y)) \nabla u_{0}(0) \cdot \nu+\frac{\partial v_{d}^{-}}{\partial \nu_{y}}\right) \\
& \quad \times(y+\chi(y)) \mathrm{d} \sigma_{y}+O\left(\varepsilon^{3+(1 / 4)}\right) .
\end{aligned}
$$

To clarify the structure of this expression, we introduce the functions $\varphi_{j, d}, 1 \leqslant$ $j \leqslant 3$, which are solutions of the following:

$$
\left.\begin{array}{c}
\operatorname{div}_{y}\left(a_{d}(y) \nabla \varphi_{j, d}\right)=0 \quad \text { in } \tilde{\omega}, \quad \operatorname{div}\left(a_{d}(y) \nabla \varphi_{j, d}\right)=0 \quad \text { in } \mathbb{R}^{3} \backslash \tilde{\omega} \\
\varphi_{j, d} \text { is continuous across } \partial \tilde{\omega}, \\
a_{d}^{+}(y) \frac{\partial \varphi_{j, d}^{+}}{\partial \nu_{y}}-a_{d}^{-}(y) \frac{\partial \varphi_{j, d}^{-}}{\partial \nu_{y}}=\left(a_{d}^{-}-a^{-}\right)\left(\nu_{j}+\frac{\partial \chi^{j}(y)}{\partial \nu_{y}}\right) \\
\varphi_{j, d}(y) \rightarrow 0 \quad \text { when }|y| \rightarrow \infty .
\end{array}\right\}
$$

Noting that

$$
v_{d}(y)=\sum_{j=1}^{3} \varphi_{j, d}(y) \frac{\partial u_{0}}{\partial x_{j}}(0)
$$

allows us to rewrite

$u_{\varepsilon, d}(z)-u_{\varepsilon}(z)=\int_{\partial \Omega}\left(u_{\varepsilon}-u_{\varepsilon, d}\right) a_{\varepsilon} \frac{\partial G_{\varepsilon}}{\partial \nu_{x}} \mathrm{~d} \sigma_{x}+\varepsilon^{3} \nabla_{x} G_{0}(0, z) M \nabla_{x} u_{0}(0)+O\left(\varepsilon^{3+(1 / 4)}\right)$,

where the tensor $M$ is defined by

$$
M_{i j}=\int_{\partial \tilde{\omega}}\left(a^{-}(y)-a_{d}^{-}(y)\right)\left(y_{i}+\chi^{i}(y)\right)\left(\frac{\partial \varphi_{j, d}^{-}}{\partial \nu_{y}}+\left(\nu_{j}+\frac{\partial \chi^{j}(y)}{\partial \nu_{y}}\right)\right) \mathrm{d} \sigma_{y} .
$$

Using the jump condition satisfied by $\varphi_{j, d}$ across $\partial \tilde{\omega}$,

$$
\frac{\partial \varphi_{j, d}^{-}}{\partial \nu_{y}}=\frac{1}{a_{d}^{-}}\left(a_{d}^{+} \frac{\partial \varphi_{j, d}^{+}}{\partial \nu_{y}}+\left(a^{-}-a_{d}^{-}\right)\left(\nu_{j}+\frac{\partial \chi^{j}}{\partial \nu_{y}}\right)(y)\right)
$$

one sees that $M_{i j}$ can be expressed as

$$
M_{i j}=\int_{\partial \tilde{\omega}}\left(\frac{a^{-}}{a_{d}^{-}}-1\right)\left(y_{i}+\chi^{i}(y)\right)\left(a^{+}(y) \frac{\partial \varphi_{j, d}^{+}}{\partial \nu_{y}}+a^{-}(y)\left(\nu_{j}+\frac{\partial \chi^{j}(y)}{\partial \nu_{y}}\right)\right) \mathrm{d} \sigma_{y},
$$

which proves theorem 4.1 .

This formula defines a polarization tensor in the spirit of $[9,10]$. It describes the influence on the far field of a localized defect within the periodic medium. One easily checks that the expression of $M_{i j}$ reduces to that given in [10] when, instead of a misplaced inclusion, one considers a defect $\left(a_{d} \neq 1\right.$ in $\left.\omega_{\varepsilon, 2}\right)$ in a homogeneous background medium ( $a$ constant and $\chi=0$ ). Also, adapting the proof of $[10$, lemma 5] shows that $M$ is symmetric. 


\section{Appendix A. Proofs of the estimates}

The proofs of theorems $3.4-3.7$ and of lemma 3.5 are collected in this appendix.

\section{A.1. Proof of theorem 3.4}

The proof of this result is based on two main ingredients. The first is the 'threestep compactness method' of Avellaneda and Lin [4,5], who proved Hölder and Lipschitz estimates on $u_{\varepsilon}$, when the coefficients of $L_{\varepsilon}$ are smooth (Hölder continuous). The second is the Hölder regularity results for the gradients in composite media containing inclusions with $\mathcal{C}^{1, \alpha}$-regular boundaries $[15,16]$. Theorem 3.4 generalizes theorem 3.3 to non-zero right-hand side. Its proof closely follows that of theorem 3.3 (in [15, theorem 0.2]), which itself is based on the arguments of $[4,5]$.

In the sequel, for each $r, 0<r<1$, and $x \in \mathbb{R}^{3}$, we set

$$
\begin{gathered}
B(x, r)=\left\{y \in \mathbb{R}^{3} /|x-y|<r\right\}, \quad B_{r}=B(0, r), \\
f_{D} f=\frac{1}{|D|} \int_{D} f, \quad\left(\bar{u}_{\varepsilon}\right)_{x, r}=f_{B(x, r)} u_{\varepsilon} .
\end{gathered}
$$

We recall the classical characterization of Hölder spaces [8] in terms of the seminorm

$$
[u]_{\mathcal{C}^{0, \alpha}(\bar{\Omega})}=\sup _{x, x^{\prime} \in \Omega} \frac{\left|u(x)-u\left(x^{\prime}\right)\right|}{\left|x-x^{\prime}\right|^{\alpha}} .
$$

For each $0<\alpha<1$, there exist positive constants $c_{1}, c_{2}$, which depend only on $\Omega$ and $\alpha$ such that, for all $u \in \mathcal{C}^{0, \alpha}(\bar{\Omega})$,

$$
c_{1}[u]_{\mathcal{C}^{0, \alpha}(\bar{\Omega})} \leqslant \sup _{x \in \Omega} \sup _{r>0}\left[\frac{1}{r^{2 \alpha}} f_{\Omega \cap B(x, r)}\left(u-(\bar{u})_{x, r}\right)^{2}\right]^{1 / 2} \leqslant c_{2}[u]_{\mathcal{C}^{0, \alpha}(\bar{\Omega})} .
$$

We assume that the coefficient $a$ is piecewise smooth and that the boundaries of the inclusions have regularity $\mathcal{C}^{1, \alpha}$ for some $0<\alpha<1$. Let $\mu=\alpha / 2(\alpha+1)$. We begin by proving interior Hölder estimates on $u_{\varepsilon}$ (see [15, theorem 5.1]).

TheOREM A.1 (interior Hölder estimates). Let $f \in L^{\infty}\left(B_{1}\right)$ and $h \in \mathcal{C}^{0, \mu}\left(B_{1}\right)^{3}$. We then assume that $u_{\varepsilon}$ satisfies

$$
L_{\varepsilon} u_{\varepsilon}=f+\varepsilon \operatorname{div}\left(b_{\varepsilon} h\right) \quad \text { in } B_{1} \text {. }
$$

There exists a constant $C$, which depends only on $\mu, \lambda, \Lambda$ and $\alpha$, but which is independent of $\varepsilon$ and of the distances between the inclusions, such that

$$
\left\|u_{\varepsilon}\right\|_{\mathcal{C}^{0, \mu}\left(B_{1 / 2}\right)} \leqslant C\left(\left\|u_{\varepsilon}\right\|_{L^{2}\left(B_{1}\right)}+\|f\|_{L^{\infty}\left(B_{1}\right)}+\|h\|_{\mathcal{C}^{0, \mu}\left(B_{1}\right)}\right) .
$$

The theorem results from the following three lemmas. The difference with [4] mainly lies in the proof of the third lemma, where the regularity hypotheses on the conductivity are determinant. 
Lemma A.2 (one-step improvement). There exist $\theta>0$ and $0<\varepsilon_{0}<1$, which depend only on $\mu, \alpha, \lambda$ and $\Lambda$, such that if $u_{\varepsilon}, f$ and $h$ satisfy

$$
\begin{gathered}
L_{\varepsilon} u_{\varepsilon}=f+\varepsilon \operatorname{div}\left(b_{\varepsilon} h\right) \quad \text { in } B_{1}, \\
f_{B_{1}}\left|u_{\varepsilon}\right|^{2} \leqslant 1, \\
\|f\|_{L^{\infty}\left(B_{1}\right)} \leqslant \varepsilon_{0}, \\
\|h\|_{\mathcal{C}^{0, \mu}\left(B_{1}\right)} \leqslant \varepsilon_{0},
\end{gathered}
$$

then, for $0<\varepsilon \leqslant \varepsilon_{0}$,

$$
f_{B_{\theta}}\left|u_{\varepsilon}-\left(\bar{u}_{\varepsilon}\right)_{0, \theta}\right|^{2} \leqslant \theta^{2 \mu}
$$

Proof. Let $\mu<\mu^{\prime}<1$. As the homogenized operator $L_{0}$ is elliptic with constant coefficients, solutions to $-\operatorname{div}\left(A \nabla u_{0}\right)=0$ in $B_{1}$ are smooth. In particular, there exists $0<\theta<1$ such that

$$
f_{B_{\theta}}\left(u_{0}-\left(\overline{u_{0}}\right)_{0, \theta}\right)^{2} \leqslant \theta^{2 \mu^{\prime}} f_{B_{1}} u_{0}^{2}
$$

We fix a value of $\theta$ for which (A 4) holds. We prove (A 3) by contradiction, as follows. Assume that there is a sequence $L_{\varepsilon_{j}}^{j}, u_{\varepsilon_{j}}, f_{\varepsilon_{j}}, h_{\varepsilon_{j}}$ that satisfies

$$
L_{\varepsilon_{j}}^{j} u_{\varepsilon_{j}}=f_{\varepsilon_{j}}+\varepsilon_{j} \operatorname{div}\left(b_{\varepsilon_{j}} h_{\varepsilon_{j}}\right) \text { in } B_{1},
$$

with

$$
f_{B_{1}} u_{\varepsilon_{j}}^{2} \leqslant 1 \quad \text { and } \quad \lim \left\|f_{\varepsilon_{j}}\right\|_{L^{\infty}\left(B_{1}\right)}=\lim \left\|h_{\varepsilon_{j}}\right\|_{\mathcal{C}^{0, \mu}\left(B_{1}\right)}=\lim \varepsilon_{j}=0
$$

and such that

$$
f_{B_{\theta}}\left|u_{\varepsilon_{j}}-\left(u_{\varepsilon_{j}}^{-}\right)_{0, \theta}\right|^{2}>\theta^{2 \mu}
$$

Extracting a subsequence, we find an operator $L_{0}$, limit of the operators $L_{\varepsilon_{j}}^{j}$ in the sense of homogenization, and a function $u_{0} \in H_{\mathrm{loc}}^{1}\left(B_{1}\right)$, such that

$$
\begin{aligned}
& u_{\varepsilon_{j}} \rightarrow u_{0} \quad \text { weakly in } L^{2}\left(B_{1}\right), \\
& u_{\varepsilon_{j}} \rightarrow u_{0}
\end{aligned}
$$

As $f_{\varepsilon_{j}}+\varepsilon_{j} \operatorname{div}\left(b_{\varepsilon_{j}} h_{\varepsilon_{j}}\right)$ converges to 0 strongly in $H^{-1}\left(B_{1}\right)$, we see that $L_{0}\left(u_{0}\right)=0$ in $B_{1}$. Taking limits in (A 5) we get

$$
\theta^{2 \mu} \leqslant f_{B_{\theta}}\left|u_{0}-\left(\overline{u_{0}}\right)_{0, \theta}\right|^{2} \leqslant \theta^{2 \mu^{\prime}},
$$

which is a contradiction. Hence, (A 3) holds for some $\varepsilon_{0}>0$.

Lemma A.3 (iteration). Let $\theta$ and $\varepsilon_{0}$ be as in lemma A.2. Then, for all $u_{\varepsilon} \in$ $L^{2}\left(B_{1}\right), f \in L^{\infty}\left(B_{1}\right)$ and $h \in \mathcal{C}^{0, \mu}\left(B_{1}\right)$ that satisfy

$$
L_{\varepsilon} u_{\varepsilon}=f+\varepsilon \operatorname{div}\left(b_{\varepsilon} h\right) \text { in } B_{1} \text {, }
$$


and for all $k \geqslant 1$ such that $\varepsilon / \theta^{k} \leqslant \varepsilon_{0}$,

$$
f_{B_{\theta^{k}}}\left|u_{\varepsilon}-\left(\bar{u}_{\varepsilon}\right)_{0, \theta^{k}}\right|^{2} \leqslant \theta^{2 k \mu}\left[\left(f_{B_{1}}\left|u_{\varepsilon}\right|^{2}\right)^{1 / 2}+\frac{1}{\varepsilon_{0}}\left(\left\|f_{\varepsilon}\right\|_{L^{\infty}\left(B_{1}\right)}+\|h\|_{\mathcal{C}^{0, \alpha}\left(B_{1}\right)}\right)\right]^{2} .
$$

Proof. The proof is by induction on $k$. Lemma A.2 shows that (A 6) holds for $k=1$. Let

$$
J=\left[\left(f_{B_{1}}\left|u_{\varepsilon}\right|^{2}\right)^{1 / 2}+\frac{1}{\varepsilon_{0}}\left(\|f\|_{L^{\infty}\left(B_{1}\right)}+\|h\|_{\mathcal{C}^{0, \mu}\left(B_{1}\right)}\right)\right]^{2} .
$$

and, for $k$ satisfying $\varepsilon / \theta^{k} \leqslant \varepsilon_{0}$ and $x \in B_{1}$, let

$$
w_{\varepsilon}(x)=J^{-1} \theta^{-k \mu}\left[u_{\varepsilon}\left(\theta^{k} x\right)-\left(\bar{u}_{\varepsilon}\right)_{0, \theta^{k}}\right] .
$$

Then $w_{\varepsilon}$ solves

$$
L_{\varepsilon / \theta^{k}} w_{\varepsilon}=\hat{f}_{\varepsilon}+\frac{\varepsilon}{\theta^{k}} \operatorname{div}\left(b_{\varepsilon / \theta^{k}} \hat{h}_{\varepsilon}\right),
$$

where, for $x \in B_{1}, \hat{f}_{\varepsilon}(x)=J^{-1} \theta^{k(2-\mu)} f\left(\theta^{k} x\right)$ and $\hat{h}_{\varepsilon}(x)=J^{-1} \theta^{k(2-\mu)} h\left(\theta^{k} x\right)$. One may easily check that

$$
\begin{aligned}
\left\|\hat{f}_{\varepsilon}\right\|_{L^{\infty}\left(B_{1}\right)} & \leqslant J^{-1} \theta^{k(2-\mu)}\|f\|_{L^{\infty}\left(B_{1}\right)} \leqslant \varepsilon_{0}, \\
\left\|\hat{h}_{\varepsilon}\right\|_{\mathcal{C}^{0, \mu}\left(B_{1}\right)} & \leqslant J^{-1} \theta^{k(2-\mu)}\|h\|_{\mathcal{C}^{0, \mu}\left(B_{1}\right)} \leqslant \varepsilon_{0} .
\end{aligned}
$$

By the induction hypothesis, we see that

$$
f_{B_{1}}\left|w_{\varepsilon}(x)\right|^{2} \leqslant 1
$$

Thus, we can apply lemma A.2: $w_{\varepsilon}$ satisfies (A 3) which, expressed in terms of $u_{\varepsilon}$, yields (A 6).

Lemma A.4 (blow-up). Assume that $u_{\varepsilon} \in L^{2}\left(B_{1}\right), f \in L^{\infty}\left(B_{1}\right)$ and $h \in \mathcal{C}^{0, \mu}\left(B_{1}\right)$ satisfy

$$
L_{\varepsilon} u_{\varepsilon}=f+\varepsilon \operatorname{div}\left(b_{\varepsilon} h\right) \text { in } B_{1} .
$$

There then exists a constant $C$, that depends only on $\mu, \lambda, \Lambda$ and the regularity of the dividing interfaces, such that

$$
\left\|u_{\varepsilon}\right\|_{\mathcal{C}^{0, \mu}\left(B_{1 / 2}\right)} \leqslant C\left(\left\|u_{\varepsilon}\right\|_{L^{2}\left(B_{1}\right)}+\|f\|_{L^{\infty}\left(B_{1}\right)}+\|h\|_{\mathcal{C}^{0, \mu}\left(B_{1}\right)}\right) .
$$

Proof. In view of (A 1), we need only prove that

$$
f_{B(x, r)}\left|u_{\varepsilon}-\left(\bar{u}_{\varepsilon}\right)_{x, r}\right|^{2} \leqslant C r^{2 \mu}\left(\left\|u_{\varepsilon}\right\|_{L^{2}\left(B_{1}\right)}+\|f\|_{L^{\infty}\left(B_{1}\right)}+\|h\|_{\mathcal{C}^{0, \mu}\left(B_{1}\right)}\right)^{2},
$$

for all $0<r \leqslant \frac{1}{4}$ and $|x|<\frac{1}{2}$. We establish (A 9) for $x=0$. By lemma A.3, (A 9) with $x=0$ holds for $r \geqslant \varepsilon / \varepsilon_{0}$. For $y \in B_{2 / \varepsilon_{0}}$, let

$$
w_{\varepsilon}(y)=\varepsilon^{-\mu}\left(u_{\varepsilon}(\varepsilon y)-\left(\bar{u}_{\varepsilon}\right)_{0,2 \varepsilon / \varepsilon_{0}}\right) .
$$


By applying (A 9) with $r=2 \varepsilon / \varepsilon_{0}$, we show that

$$
\begin{aligned}
\left\|w_{\varepsilon}\right\|_{L^{2}\left(B_{2 / \varepsilon_{0}}\right)}^{2} & =\frac{\varepsilon^{-2 \mu}}{\varepsilon_{0}^{3}} f_{B_{2 \varepsilon / \varepsilon_{0}}}\left(u_{\varepsilon}(x)-\left(\bar{u}_{\varepsilon}\right)_{0,2 \varepsilon / \varepsilon_{0}}\right)^{2} \mathrm{~d} x \\
& \leqslant \frac{\varepsilon^{-2 \mu}}{\varepsilon_{0}^{3}}\left(\frac{2 \varepsilon}{\varepsilon_{0}}\right)^{2 \mu} J^{2} \\
& \leqslant C J^{2}
\end{aligned}
$$

with $J$ as in (A 7), so that $w_{\varepsilon}$ is uniformly bounded in $L^{2}\left(B_{2 / \varepsilon_{0}}\right)$. Moreover, $w_{\varepsilon}$ solves the following equation, where the operator and the domain (and in particular the number of inclusions) are independent of $\varepsilon$ :

$$
L_{1} w_{\varepsilon}=\hat{f}_{\varepsilon}+\operatorname{div}\left(b_{1} \hat{h}_{\varepsilon}\right) \quad \text { in } B_{2 / \varepsilon_{0}}
$$

with $\hat{f}_{\varepsilon}(x)=\varepsilon^{2-\mu} f(\varepsilon x)$ and $\hat{h}_{\varepsilon}(x)=\varepsilon^{2-\mu} h(\varepsilon x)$ for $x \in B_{2 / \varepsilon_{0}}$. We note that

$$
\left.\begin{array}{rl}
\left\|\hat{f}_{\varepsilon}\right\|_{L^{\infty}\left(B_{2 / \varepsilon_{0}}\right)} & \leqslant \varepsilon^{2-\mu}\|f\|_{L^{\infty}\left(B_{1}\right)}, \\
\left\|\hat{h}_{\varepsilon}\right\|_{\mathcal{C}^{0, \mu}\left(B_{2 / \varepsilon_{0}}\right)} & \leqslant \varepsilon^{2-\nu}\|h\|_{\mathcal{C}^{0, \mu}\left(B_{1}\right)},
\end{array}\right\}
$$

and that $b(y) \hat{h}_{\varepsilon}(y)$ has regularity $\mathcal{C}^{0, \mu}$ on each of the inclusions $\varepsilon l \bar{D}_{0}, l \in \mathbb{Z}^{3}$, contained in $B_{2 / \varepsilon_{0}}$ and has the same regularity in $B_{2 / \varepsilon_{0}} \backslash \bigcup_{\ell \in \mathbb{Z}^{3}} \ell \bar{D}_{0}$.

Therefore, we can apply the interior Hölder gradient estimates (3.13) to $w_{\varepsilon}$, in order to obtain

$$
\left\|w_{\varepsilon}\right\|_{\mathcal{C}^{0, \mu}\left(B_{1 / \varepsilon_{0}}\right)} \leqslant C\left(\left\|w_{\varepsilon}\right\|_{L^{2}\left(B_{2 / \varepsilon_{0}}\right)}+\left\|\hat{f}_{\varepsilon}\right\|_{L^{\infty}\left(B_{2 / \varepsilon_{0}}\right)}+\left\|\hat{h}_{\varepsilon}\right\|_{\mathcal{C}^{0, \mu}\left(B_{2 / \varepsilon_{0}}\right)}\right) .
$$

Thus, for all $s \leqslant 1 / \varepsilon_{0}$, we have

$$
\begin{aligned}
\varepsilon^{-2 \mu} f_{B_{\varepsilon s}} & \left|u_{\varepsilon}(x)-\left(\bar{u}_{\varepsilon}\right)_{0, \varepsilon s}\right|^{2} \mathrm{~d} x \\
\quad & =f_{B_{s}}\left|w_{\varepsilon}-\left(\bar{w}_{\varepsilon}\right)_{0, s}\right|^{2} \\
& \leqslant C s^{2 \mu}\left(\left\|w_{\varepsilon}\right\|_{L^{2}\left(B_{2 / \varepsilon_{0}}\right)}+\left\|\hat{f}_{\varepsilon}\right\|_{L^{\infty}\left(B_{2 / \varepsilon_{0}}\right)}+\left\|\hat{h}_{\varepsilon}\right\|_{\mathcal{C}^{0, \mu}\left(B_{2 / \varepsilon_{0}}\right)}\right)^{2} .
\end{aligned}
$$

Setting $r=s \varepsilon$ and combining this last identity with (A 12), (A 10) and (A 13), we finally obtain

$$
f_{B_{r}}\left|u_{\varepsilon}-\left(\bar{u}_{\varepsilon}\right)_{0, r}\right|^{2} \leqslant C r^{2 \nu}\left(\left\|u_{\varepsilon}\right\|_{L^{2}\left(B_{1}\right)}+\|f\|_{L^{\infty}\left(B_{1}\right)}+\|h\|_{\mathcal{C}^{0, \mu}\left(B_{1}\right)}\right)^{2},
$$

which is (A 9) at $x=0$. By translation, this estimate remains true for all $x \in B_{1 / 2}$. The lemma (and theorem A.1) is proved.

Let $\chi$ be the cell function defined in (3.4). To prove theorem 3.4, we again apply the three-step method, this time to estimate the quantity

$$
\left\|u_{\varepsilon}(x)-u_{\varepsilon}(0)-\left(x+\varepsilon \chi\left(\frac{x}{\varepsilon}\right)\right)\left(\overline{\nabla u_{\varepsilon}}\right)_{0, \theta}\right\|_{L^{\infty}\left(B_{\theta}\right)} .
$$


LemMA A.5 (one-step improvement). There exist $0<\theta, \varepsilon_{0}<1$ which depend only on $\lambda, \Lambda, \mu$ and $\alpha$, such that, if $u_{\varepsilon}, f$ and $h$ satisfy

$$
L_{\varepsilon} u_{\varepsilon}=f+\varepsilon \operatorname{div}\left(b_{\varepsilon} h\right) \quad \text { in } B_{1}
$$

with $\left\|u_{\varepsilon}\right\|_{L^{\infty}\left(B_{1}\right)} \leqslant 1,\|f\| L^{\infty}\left(B_{1}\right) \leqslant 1$ and $\|h\|_{\mathcal{C}^{0, \mu}\left(B_{1}\right)} \leqslant 1$ then, for $0<\varepsilon \leqslant \varepsilon_{0}$,

$$
\sup _{|x|<\theta}\left|u_{\varepsilon}(x)-u_{\varepsilon}(0)-\left(x+\varepsilon \chi\left(\frac{x}{\varepsilon}\right)\right)\left(\overline{\nabla u_{\varepsilon}}\right)_{0, \theta}\right| \leqslant \theta^{1+\mu / 2} .
$$

Proof. Let $\mu<\mu^{\prime}<1$. Recalling (3.8), let $u_{0}$ and $f_{0}$ satisfy

$$
L_{0} u_{0}=f_{0} \quad \text { in } B_{1}
$$

Classical regularity estimates $[11]$ show that $u_{0} \in \mathcal{C}^{1, \mu}(\bar{\Omega})$. Thus, there exists $0<$ $\theta<1$, which depends only on $\lambda$ and $\Lambda$, such that

$$
\sup _{|x|<\theta}\left|u_{0}(x)-u_{0}(0)-x\left(\nabla \overline{u_{0}}\right)_{0, \theta}\right| \leqslant \theta^{1+\mu^{\prime} / 2}\left(\left\|u_{0}\right\|_{L^{\infty}\left(B_{1}\right)}+\left\|f_{0}\right\|_{L^{\infty}\left(B_{1}\right)}\right) .
$$

By fixing this value of $\theta$, we prove (A 14) by contradiction.

Suppose on the contrary that there is a sequence $\varepsilon_{j} \rightarrow 0$ and sequences $L_{\varepsilon_{j}}^{j}$, $u_{\varepsilon_{j}}, f_{\varepsilon_{j}}$ and $h_{\varepsilon_{j}}$, such that

$$
L_{\varepsilon_{j}}^{j} u_{\varepsilon_{j}}=f_{\varepsilon_{j}}+\varepsilon_{j} \operatorname{div}\left(b_{\varepsilon_{j}} h_{\varepsilon_{j}}\right) \quad \text { in } B_{1}
$$

and

$$
\begin{aligned}
\left\|u_{\varepsilon_{j}}\right\|_{L^{\infty}\left(B_{1}\right)} & \leqslant 1, \\
\left\|f_{\varepsilon_{j}}\right\|_{L^{\infty}\left(B_{1}\right)} & \leqslant 1, \\
\left\|h_{\varepsilon_{j}}\right\|_{\mathcal{C}^{0, \mu}\left(B_{1}\right)} & \leqslant 1,
\end{aligned}
$$

and for which

$$
\sup _{|x|<\theta}\left|u_{\varepsilon_{j}}(x)-u_{\varepsilon_{j}}(0)-\left(x+\varepsilon_{j} \chi\left(\frac{x}{\varepsilon_{j}}\right)\right)\left(\overline{\nabla u_{\varepsilon_{j}}}\right)_{0, \theta}\right|>\theta^{1+\mu / 2} .
$$

Passing to a subsequence (not renamed) and using theorem A.1, we find an operator $L_{0}$ and functions $u_{0} \in H_{\mathrm{loc}}^{1}\left(B_{1}\right)$ and $f_{0} \in L^{\infty}\left(B_{1}\right)$, such that

$$
\begin{aligned}
& f_{\varepsilon_{j}} \rightarrow f_{0} \text { weakly in } L^{\infty}\left(B_{1}\right), \quad u_{\varepsilon_{j}} \rightarrow u_{0} \text { uniformly in } B_{\theta}, \\
& u_{\varepsilon_{j}} \rightarrow u_{0} \text { weakly-* in } L^{\infty}\left(B_{1}\right), \quad \nabla u_{\varepsilon_{j}} \rightarrow \nabla u_{0} \text { weakly in } L^{2}\left(B_{\theta}\right) \text {. }
\end{aligned}
$$

We also note that $\varepsilon_{j} \operatorname{div}\left(b_{\varepsilon_{j}} h_{\varepsilon_{j}}\right) \rightarrow 0$ strongly in $H^{-1}\left(B_{1}\right)$, such that $L_{0} u_{0}=f_{0}$ in $B_{1}$.

Before passing to the limit in (A 16), we show that $\left|\left(\overline{\nabla u_{\varepsilon_{j}}}\right)_{0, \theta}\right|$ is uniformly bounded by a constant that depends only on $\theta$. Indeed, let $v \in \mathcal{C}_{0}^{\infty}\left(B_{(1+\theta) / 2}\right)$ satisfy 
$0 \leqslant v \leqslant 1$ and $v \equiv 1$ on $B_{\theta}$. We have

$$
\begin{aligned}
\left|\left(\overline{\nabla u_{\varepsilon_{j}}}\right)_{0, \theta}\right| & =\left|\frac{1}{\left|B_{\theta}\right|} \int_{B_{\theta}} \nabla u_{\varepsilon_{j}}\right| \\
& \leqslant \frac{1}{\left|B_{\theta}\right|^{1 / 2}}\left(\int_{B_{\theta}}\left|\nabla\left(u_{\varepsilon_{j}}\right)\right|^{2}\right)^{1 / 2} \\
& \leqslant \frac{1}{\left|B_{\theta}\right|^{1 / 2}}\left(\int_{B_{1}}\left|\nabla\left(v u_{\varepsilon_{j}}\right)\right|^{2}\right)^{1 / 2} .
\end{aligned}
$$

One can easily check that

$$
\begin{aligned}
\int_{B_{1}}\left|\nabla\left(v u_{\varepsilon_{j}}\right)\right|^{2} & =\int_{B_{1}} \nabla u_{\varepsilon_{j}} \cdot \nabla\left(v^{2} u_{\varepsilon_{j}}\right)+\int_{B_{1}} u_{\varepsilon_{j}}^{2}|\nabla v|^{2} \\
& \leqslant \int_{B_{1}} f_{\varepsilon_{j}} v^{2} u_{\varepsilon_{j}}-\varepsilon_{j} \int_{B_{1}} b_{\varepsilon_{j}} h_{\varepsilon_{j}} \cdot \nabla\left(v^{2} u_{\varepsilon_{j}}\right)+\int_{B_{1}} u_{\varepsilon_{j}}^{2}|\nabla v|^{2} .
\end{aligned}
$$

Therefore, given the uniform bounds on $u_{\varepsilon_{j}}$ and $f_{\varepsilon_{j}}$, we conclude that

$$
\begin{gathered}
\left|\left(\overline{\nabla u_{\varepsilon_{j}}}\right)_{0, \theta}\right| \leqslant C(\theta), \\
\sup _{|x|<\theta}\left|\varepsilon_{j} \chi\left(\frac{x}{\varepsilon_{j}}\right)\left(\overline{\nabla u_{\varepsilon_{j}}}\right)_{0, \theta}\right| \leqslant \varepsilon_{j} C(\theta) \rightarrow 0 .
\end{gathered}
$$

Returning to (A 16), and passing to the limit $\varepsilon_{j} \rightarrow 0$ yields

$$
\theta^{1+\mu / 2} \leqslant \sup _{|x|<\theta}\left|u_{0}(x)-u_{0}(0)-x\left(\nabla \overline{u_{0}}\right)_{0, \theta}\right| \leqslant \theta^{1+\mu^{\prime} / 2},
$$

which contradicts the fact that $\theta<1$.

Lemma A.6 (iteration). Let $\theta$ and $\varepsilon_{0}$ be as in lemma A.5. Suppose that $u_{\varepsilon} \in$ $L^{\infty}\left(B_{1}\right), f \in L^{\infty}\left(B_{1}\right)$ and $h \in \mathcal{C}^{0, \mu}\left(B_{1}\right)$ satisfy

$$
L_{\varepsilon} u_{\varepsilon}=f+\varepsilon \operatorname{div}\left(b_{\varepsilon} h\right) \text { in } B_{1} .
$$

Then, for all $k \geqslant 1$, with $\varepsilon / \theta^{k} \leqslant \varepsilon_{0}$, there exist $a_{k}^{\varepsilon} \in \mathbb{R}$ and $B_{k}^{\varepsilon} \in \mathbb{R}^{3}$ such that

$$
\begin{aligned}
& \left|a_{k}^{\varepsilon}\right| \leqslant C_{1}\left(\left\|u_{\varepsilon}\right\|_{L^{\infty}\left(B_{1}\right)}+\|f\|_{L^{\infty}\left(B_{1}\right)}+\|h\|_{\mathcal{C}^{0, \mu}\left(B_{1}\right)}\right) \\
& \left|B_{k}^{\varepsilon}\right| \leqslant C_{2}\left(1+\sum_{j=1}^{k} \theta^{j \mu / 2}\right)\left(\left\|u_{\varepsilon}\right\|_{L^{\infty}\left(B_{1}\right)}+\|f\|_{L^{\infty}\left(B_{1}\right)}+\|h\|_{\mathcal{C}^{0, \mu}\left(B_{1}\right)}\right),
\end{aligned}
$$

and such that

$$
\begin{aligned}
\sup _{|x|<\theta^{k}} \mid u_{\varepsilon}(x)-u_{\varepsilon}(0) & -\varepsilon a_{k}^{\varepsilon}-\left[x+\varepsilon \chi\left(\frac{x}{\varepsilon}\right)\right] B_{k}^{\varepsilon} \mid \\
& \leqslant \theta^{k(1+\mu / 2)}\left(\left\|u_{\varepsilon}\right\|_{L^{\infty}\left(B_{1}\right)}+\|f\|_{L^{\infty}\left(B_{1}\right)}+\|h\|_{\mathcal{C}^{0, \mu}\left(B_{1}\right)}\right) .
\end{aligned}
$$

Here $C_{1}$ and $C_{2}$ are generic constants, which depend only on $\theta, \varepsilon_{0}, \lambda$ and $\Lambda$. 
Proof. We set

$$
J=\left(\left\|u_{\varepsilon}\right\|_{L^{\infty}\left(B_{1}\right)}+\|f\|_{L^{\infty}\left(B_{1}\right)}+\|h\|_{\mathcal{C}^{0, \mu}\left(B_{1}\right)}\right) .
$$

The proof is by induction on $k$. By lemma A.5, estimate (A 19) holds for $k=1$, with $a_{1}^{\varepsilon}=0$ and $B_{1}^{\varepsilon}=\left(\overline{\nabla u_{\varepsilon}}\right)_{0, \theta}$.

Suppose that (A 19) holds for some $k$ such that $\varepsilon / \theta^{k} \leqslant \varepsilon_{0}$. For $|x|<1$ let

$$
w_{\varepsilon}(x)=\theta^{-k(1+\mu / 2)} J^{-1}\left(u_{\varepsilon}\left(\theta^{k} x\right)-u_{\varepsilon}(0)-\varepsilon a_{k}^{\varepsilon}-\left[\theta^{k} x+\varepsilon \chi\left(\frac{\theta^{k} x}{\varepsilon}\right)\right] B_{k}^{\varepsilon}\right) .
$$

This function solves

$$
L_{\varepsilon / \theta^{k}} w_{\varepsilon}=\hat{f}_{\varepsilon}+\frac{\varepsilon}{\theta^{k}} \operatorname{div}\left(b_{\varepsilon / \theta^{k}} \hat{h}_{\varepsilon}\right) \quad \text { in } B(0,1),
$$

where, for $x \in B_{1}, \hat{f}_{\varepsilon}(x)=\theta^{k(1-\mu / 2)} J^{-1} f\left(\theta^{k} x\right)$ and $\hat{h}_{\varepsilon}(x)=\theta^{k(1-\mu / 2)} J^{-1} h\left(\theta^{k} x\right)$. One may easily see that $\left\|\hat{f}_{\varepsilon}\right\|_{L^{\infty}\left(B_{1}\right)} \leqslant 1$ and $\left\|\hat{h}_{\varepsilon}\right\|_{\mathcal{C}^{0, \mu}\left(B_{1}\right)} \leqslant 1$. By the induction hypotheses (A 19), $\left\|w_{\varepsilon}\right\|_{L^{\infty}\left(B_{1}\right)} \leqslant 1$, so that, on applying lemma A.5 to $w_{\varepsilon}$, we get

$$
\sup _{|x|<\theta}\left|w_{\varepsilon}(x)-w_{\varepsilon}(0)-\left[x+\frac{\varepsilon}{\theta^{k}} \chi\left(\frac{\theta^{k} x}{\varepsilon}\right)\right]\left(\overline{\nabla w_{\varepsilon}}\right)_{0, \theta}\right| \leqslant \theta^{1+\mu / 2} .
$$

Rewriting this inequality in terms of $u_{\varepsilon}$, we obtain

$$
\begin{array}{r}
\sup _{|x|<\theta}\left|u_{\varepsilon}\left(\theta^{k} x\right)-u_{\varepsilon}(0)+\varepsilon \chi(0) B_{k}^{\varepsilon}-\left[\theta^{k} x+\varepsilon \chi\left(\frac{\theta^{k} x}{\varepsilon}\right)\right]\left(B_{k}^{\varepsilon}+J \theta^{k \mu / 2}\left(\overline{\nabla w_{\varepsilon}}\right)_{0, \theta}\right)\right| \\
\leqslant J \theta^{(k+1)(1+\mu / 2)} .
\end{array}
$$

If we set $a_{k+1}^{\varepsilon}=-\chi(0) B_{k}^{\varepsilon}, B_{k+1}^{\varepsilon}=B_{k}^{\varepsilon}+J \theta^{k \mu / 2}\left(\overline{\nabla w_{\varepsilon}}\right)_{0, \theta}$, substitute these expressions in (A 21), and make the change of variables $\theta^{k} x \rightarrow x$, we obtain (A 19) with $k+1$ instead of $k$. Moreover, as in the proof of lemma A.5,

$$
\left|\left(\overline{\nabla w_{\varepsilon}}\right)_{0, \theta}\right| \leqslant C
$$

where $C$ depends on $\theta$ but not on $\varepsilon$ or $k$ and, given the initial choice of $a_{1}^{\varepsilon}$ and $B_{1}^{\varepsilon}$, it is easy to check that the sequences $\left\{a_{k}^{\varepsilon}\right\}$ and $\left\{B_{k}^{\varepsilon}\right\}$ satisfy the estimates (A 17) and (A 18). Lemma A.6 is thus proved.

Proof of theorem 3.4. Let $k \in \mathbb{N}$ be such that $\varepsilon / \theta^{k} \leqslant \varepsilon_{0} \leqslant \varepsilon / \theta^{k+1}$. By lemma A.6 and by recalling (A 20),

$$
\sup _{|x|<\varepsilon / \varepsilon_{0}}\left|u_{\varepsilon}(x)-u_{\varepsilon}(0)-\varepsilon a_{k}^{\varepsilon}-\left(x+\varepsilon \chi\left(\frac{x}{\varepsilon}\right)\right) B_{k}^{\varepsilon}\right| \leqslant \theta^{k(1+\mu / 2)} J .
$$

Invoking the estimates (A 17) and (A 18) and rescaling the above inequality, we get

$$
\sup _{|x|<1 / \varepsilon_{0}}\left|\frac{u_{\varepsilon}(\varepsilon x)-u_{\varepsilon}(0)}{\varepsilon}\right| \leqslant\left|a_{k}^{\varepsilon}-[x+\chi(x)] B_{k}^{\varepsilon}\right|+\varepsilon_{0} \frac{\theta^{k(1+\mu / 2)}}{\theta^{k+1}} J \leqslant C J .
$$

For $|x|<1 / \varepsilon$, the function

$$
v_{\varepsilon}(x)=\frac{u_{\varepsilon}(\varepsilon x)-u_{\varepsilon}(0)}{\varepsilon},
$$


satisfies

$$
L_{1} v_{\varepsilon}=\hat{f}_{\varepsilon}(x)+\operatorname{div}\left(b_{1}(y) \hat{h}_{\varepsilon}\right) \quad \text { in } B_{1 / \varepsilon_{0}},
$$

where $\hat{f}_{\varepsilon}(x)=\varepsilon f(\varepsilon x)$ and $\hat{h}_{\varepsilon}(x)=\varepsilon h(\varepsilon x), x \in B_{1 / \varepsilon_{0}}$, and

$$
\begin{aligned}
\left\|v_{\varepsilon}\right\|_{L^{\infty}\left(B_{1 / \varepsilon_{0}}\right)} & \leqslant C J, \\
\left\|\hat{f}_{\varepsilon}\right\|_{L^{\infty}\left(B_{1 / \varepsilon_{0}}\right)} & \leqslant \varepsilon J, \\
\left\|\hat{h}_{\varepsilon}\right\|_{\mathcal{C}^{0, \mu}\left(B_{1 / \varepsilon_{0}}\right)} & \leqslant \varepsilon J .
\end{aligned}
$$

In the above equation, neither the operator nor the domain depend on $\varepsilon$. We again remark that the function $b \hat{h}_{\varepsilon}$ has regularity $\mathcal{C}^{0, \mu}$ on each of the inclusions contained in $B_{1 / \varepsilon_{0}}$ and on the complementary of the inclusions in $B_{1 / \varepsilon_{0}}$. We can therefore apply the interior gradient estimate $(3.14)$ to $v_{\varepsilon}$, to obtain

$$
\left\|\nabla v_{\varepsilon}\right\|_{L^{\infty}\left(B_{1 / 2 \varepsilon_{0}}\right)} \leqslant C\left(\left\|v_{\varepsilon}\right\|_{L^{\infty}\left(B_{1 / \varepsilon_{0}}\right)}+\left\|\hat{f}_{\varepsilon}\right\|_{L^{\infty}\left(B_{1 / \varepsilon_{0}}\right)}+\left\|\hat{h}_{\varepsilon}\right\|_{\mathcal{C}^{0, \mu}\left(B_{1 / \varepsilon_{0}}\right)}\right),
$$

which shows that

$$
\left\|\nabla u_{\varepsilon}\right\|_{L^{\infty}\left(B_{\varepsilon / 2 \varepsilon_{0}}\right)} \leqslant C J .
$$

The same estimate can be established in $B\left(x, \varepsilon / 2 \varepsilon_{0}\right)$ for any $x \in B_{1 / 2}$. The proof of theorem 3.4 follows by combining this estimate with (A 2).

\section{A.2. Proof of lemma 3.5}

Error estimates in $L^{2}$ between $u_{\varepsilon}$ and $u_{0}$ are well known for Dirichlet problems. For Neumann boundary conditions, Moskow and Vogelius [20] derived twodimensional estimates, using the fact that the harmonic conjugate of the potential satisfies Dirichlet boundary conditions. Our proof does not use this property, although we follow the structure of Moskow and Vogelius's argument.

STEP 1. We transform the equation into a first-order system

$$
\begin{array}{r}
a_{\varepsilon} \nabla u_{\varepsilon}-v_{\varepsilon}=0, \\
-\operatorname{div}\left(v_{\varepsilon}\right)=0 .
\end{array}
$$

and seek an asymptotic expansion for both $u_{\varepsilon}$ and $v_{\varepsilon}$. Such an expansion is given explicitly in three dimensions, in [6, pp. 58-65]. Recalling the notation of $\S 3.1$, one may easily check that the first term in the expansion of $u_{\varepsilon}$ must be the potential $u_{0}$ of (3.8) and that

$$
\left.\begin{array}{rl}
-\operatorname{div}_{y} v_{0} & =0, \\
a(y) \nabla_{y} u_{1}+a(y) \nabla_{x} u_{0}-v_{0} & =0,
\end{array}\right\}
$$

Denoting by $e_{p}, 1 \leqslant p \leqslant 3$, the canonical basis of $\mathbb{R}^{3}$, we set

$$
u_{1}(x, y)=-\chi_{j}(y) \frac{\partial u_{0}}{\partial x_{j}}
$$


and we define the functions $\tilde{\chi}_{p} \in H_{\#}^{1}(Y)^{3}$ by

$$
\left.\begin{array}{rl}
\operatorname{curl}_{y}\left(a^{-1}(y) \operatorname{curl}_{y}\left(\tilde{\chi}_{p}\right)\right) & =\operatorname{curl}_{y}\left(a^{-1}(y) e_{p}\right), \\
\operatorname{div}_{y}\left(\tilde{\chi}_{p}\right) & =0, \\
f_{Y} \tilde{\chi}_{p} & =0 .
\end{array}\right\}
$$

These functions are related to the usual correctors $\chi_{j}$ defined in (3.4) by

$$
a^{-1}(y)\left(I-\operatorname{curl}_{y} \tilde{\chi}\right)=\left(I-\nabla_{y} \chi\right) A^{-1},
$$

where $\operatorname{curl}_{y} \tilde{\chi}$ and $\nabla_{y} \chi$ denote the matrices whose columns are the vectors $\operatorname{curl}_{y} \tilde{\chi}_{p}$ and $\nabla \chi_{j}$, respectively (see [6]). In particular, according to theorem 3.2 , the above relation shows that, under our hypothesis on conductivity, $\tilde{\chi} \in W^{1, \infty}(Y)^{3}$. It also shows that $\tilde{\chi}(x / \varepsilon)$ has a trace on $\partial \Omega$, which is uniformly bounded in $L^{\infty}(\partial \Omega)$. Following [6], we set

$$
\left.\begin{array}{l}
v_{0}(x, y)=\rho(x)-\operatorname{curl}_{y}\left(\tilde{\chi}_{p}(y)\right) \rho_{p}(x)=\left(I-\operatorname{curl}_{y} \tilde{\chi}\right) A \nabla u_{0}(x), \\
v_{1}(x, y)=-\operatorname{curl}_{x}\left(\tilde{\chi}_{p}(y) \rho(x)\right),
\end{array}\right\}
$$

which satisfy (A 22). ${ }^{1}$

We then form

$$
\begin{aligned}
& z_{\varepsilon}(x)=u_{\varepsilon}(x)-u_{0}(x)-\varepsilon u_{1}\left(x, \frac{x}{\varepsilon}\right), \\
& \eta_{\varepsilon}(x)=a_{\varepsilon}(x) \nabla u_{\varepsilon}(x)-v_{0}\left(x, \frac{x}{\varepsilon}\right)-\varepsilon v_{1}\left(x, \frac{x}{\varepsilon}\right),
\end{aligned}
$$

and note that $z_{\varepsilon}$ and $\eta_{\varepsilon}$ are uniformly bounded in $H^{1}(\Omega)$ and $L^{2}(\Omega)$, respectively. Since $u_{0}, u_{1}, v_{0}$ and $v_{1}$ satisfy (A 22), the proof of [19, proposition 1] shows that

$$
\begin{aligned}
\operatorname{div}\left(\eta_{\varepsilon}\right) & =0 \quad \text { in } \Omega, \\
\left\|a_{\varepsilon}(x) \nabla z_{\varepsilon}-\eta_{\varepsilon}\right\|_{L^{2}(\Omega)} & \leqslant C \varepsilon\left\|u_{0}\right\|_{H^{2}(\Omega)} .
\end{aligned}
$$

As in [20], we introduce a boundary corrector defined by

$$
\left.\begin{array}{rl}
-\operatorname{div}\left(a_{\varepsilon} \nabla B_{\varepsilon}\right) & =0 \quad \text { in } \Omega, \\
a_{\varepsilon}(x) \nabla B_{\varepsilon} \cdot \nu & =\varepsilon^{-1} \eta_{\varepsilon} \cdot \nu \quad \text { on } \partial \Omega .
\end{array}\right\}
$$

We note that, as $\eta_{\varepsilon} \in L^{2}(\Omega)$ and $\operatorname{div}\left(\eta_{\varepsilon}\right)=0$, the normal trace $\eta_{\varepsilon} \cdot \nu$ is well defined in $H^{-1 / 2}(\partial \Omega)$.

We normalize $B_{\varepsilon}$ by requiring that

$$
\int_{\Omega} \varepsilon B_{\varepsilon} \mathrm{d} \sigma=\int_{\Omega} z_{\varepsilon} \mathrm{d} \sigma=\int_{\Omega} \varepsilon u_{1} \mathrm{~d} \sigma
$$

such that

$$
\left|\int_{\Omega} \varepsilon B_{\varepsilon} \mathrm{d} x\right| \leqslant C \varepsilon\left\|u_{0}\right\|_{H^{1}(\Omega)} .
$$

${ }^{1}$ Note that $\rho(x)=f_{Y} v_{0}(x, y) \mathrm{d} y=A \nabla u_{0}(x)$. 
STEP 2. It is easy to see that [20, proposition 1] also holds in three dimensions; this states that

$$
\left\|u_{\varepsilon}(x)-u_{0}(x)-\varepsilon u_{1}\left(x, \frac{x}{\varepsilon}\right)-\varepsilon B_{\varepsilon}(x)\right\|_{H^{1}(\Omega)} \leqslant C \varepsilon\left\|u_{0}\right\|_{H^{2}(\Omega)} .
$$

STEP 3. The regularity of $u_{0}$, the boundedness of $\tilde{\chi}$, and the definition of $\eta_{\varepsilon}$ show that

$$
\left\|\eta_{\varepsilon} \cdot \nu\right\|_{L^{2}(\partial \Omega)} \leqslant C\left\|u_{0}\right\|_{H^{2}(\Omega)} .
$$

We next show an estimate on $\left\|\eta_{\varepsilon} \cdot \nu\right\|_{H^{-1}(\partial \Omega)}$. Let $\phi \in H^{1}(\partial \Omega)$ and consider the boundary-value problem

$$
\left.\begin{array}{rlrl}
-\operatorname{div}(A \nabla v) & =0 & & \text { in } \Omega, \\
v & =\phi & & \text { on } \partial \Omega .
\end{array}\right\}
$$

From the definition of $\eta_{\varepsilon}$ and from (A 24), it follows that

$$
\begin{aligned}
\int_{\partial \Omega} \eta_{\varepsilon} \cdot \nu \phi \mathrm{d} \sigma= & \int_{\Omega} \eta_{\varepsilon} \cdot \nabla v \\
= & \int_{\Omega} a_{\varepsilon} \nabla u_{\varepsilon} \cdot \nabla v-\int_{\Omega}\left(v_{0}+\varepsilon v_{1}\right) \cdot \nabla v \\
= & \int_{\Omega}\left(a_{\varepsilon} \nabla u_{\varepsilon}-A \nabla u_{0}\right) \cdot \nabla v+\int_{\Omega} \sum_{p=1}^{3} \operatorname{curl}_{y}\left(\tilde{\chi}_{p}\right)\left(\frac{x}{\varepsilon}\right) \rho_{p}(x) \cdot \nabla v \\
& \quad+\varepsilon \int_{\Omega} \operatorname{curl}_{x}(\tilde{\chi} \rho)\left(x, \frac{x}{\varepsilon}\right) \cdot \nabla v \\
= & \varepsilon \int_{\Omega} \sum_{p=1}^{3} \operatorname{curl}_{x}\left(\tilde{\chi}_{p}\left(\frac{x}{\varepsilon}\right)\right) \rho_{p}(x) \cdot \nabla v+\varepsilon \int_{\Omega} \operatorname{curl}_{x}(\tilde{\chi} \rho)\left(x, \frac{x}{\varepsilon}\right) \cdot \nabla v .
\end{aligned}
$$

Integrating by parts, we obtain

$$
\begin{aligned}
& \int_{\partial \Omega} \eta_{\varepsilon} \cdot \nu \phi \mathrm{d} \sigma=\varepsilon\left\{\int_{\Omega} \sum_{p=1}^{3} \tilde{\chi}\left(\frac{x}{\varepsilon}\right) \cdot\left(\nabla \rho_{p}(x) \times \nabla v(x)\right)\right.-\int_{\partial \Omega} \sum_{p=1}^{3}\left(\tilde{\chi}\left(\frac{x}{\varepsilon}\right) \times \nu\right) \cdot \rho_{p}(x) \nabla v(x) \mathrm{d} \sigma \\
&\left.-\int_{\partial \Omega}\left(\tilde{\chi}_{p}\left(\frac{x}{\varepsilon}\right) \rho_{p}(x) \times \nu\right) \cdot \nabla v(x) \mathrm{d} \sigma\right\} \\
&=\varepsilon\left\{\int_{\Omega} \sum_{p=1}^{3} \tilde{\chi}\left(\frac{x}{\varepsilon}\right) \cdot\left(\nabla \rho_{p}(x) \times \nabla v(x)\right)\right.-\int_{\partial \Omega} \sum_{p=1}^{3} \tilde{\chi}\left(\frac{x}{\varepsilon}\right) \cdot \rho_{p}(x)(\nu \times \nabla v(x)) \mathrm{d} \sigma \\
&\left.-\int_{\partial \Omega} \tilde{\chi}_{p}\left(\frac{x}{\varepsilon}\right) \rho_{p}(x) \cdot(\nu \times \nabla v(x)) \mathrm{d} \sigma\right\} .
\end{aligned}
$$


We remark that $\|\nabla v\|_{L^{2}(\Omega)} \leqslant C\|\phi\|_{H^{1}(\partial \Omega)}$ and that $\nu \times \nabla v$ involves only tangential derivatives of $v$; hence, $\nu \times \nabla v \in L^{2}(\partial \Omega)$ and $\|\nu \times \nabla v\|_{L^{2}(\partial \Omega)} \leqslant C\|\phi\|_{H^{1}(\partial \Omega)}$. Since $\tilde{\chi}(x / \varepsilon)$ is bounded in $L^{\infty}(\partial \Omega)$ and since $\rho(x)=A \nabla u_{0}(x)$ is regular, it follows that

$$
\int_{\partial \Omega} \eta_{\varepsilon} \cdot \nu \phi \mathrm{d} \sigma \leqslant C \varepsilon\left\|u_{0}\right\|_{H^{2}(\Omega)}\|\phi\|_{H^{1}(\partial \Omega)},
$$

in other words, $\eta_{\varepsilon} \cdot \nu_{/ \partial \Omega} \in H^{-1}(\partial \Omega)$ and

$$
\left\|\eta_{\varepsilon} \cdot \nu\right\|_{H^{-1}(\partial \Omega)} \leqslant C \varepsilon\left\|u_{0}\right\|_{H^{2}(\Omega)} .
$$

By interpolation [17, theorem 12.3], from (A 30), (A 33) we obtain

$$
\left\|a_{\varepsilon} \nabla\left(\varepsilon B_{\varepsilon}\right) \cdot \nu\right\|_{H^{-1 / 2}(\partial \Omega)}=\left\|\eta_{\varepsilon} \cdot \nu\right\|_{H^{-1 / 2}(\partial \Omega)} \leqslant C \varepsilon^{1 / 2}\left\|u_{0}\right\|_{H^{2}(\Omega)},
$$

and, thus, from standard elliptic theory we get

$$
\left\|\varepsilon B_{\varepsilon}\right\|_{H^{1}(\Omega)} \leqslant C \varepsilon^{1 / 2}\left\|u_{0}\right\|_{H^{2}(\Omega)} .
$$

STEP 4. Let $h \in L^{2}(\Omega)$, with $\int_{\Omega} h=0$, and let $w_{\varepsilon}$ denote the solution to

$$
\begin{aligned}
-\operatorname{div}\left(a_{\varepsilon} \nabla w_{\varepsilon}\right)=h & \text { in } \Omega, \\
a_{\varepsilon} \nabla w_{\varepsilon} \cdot \nu=0 & \text { on } \partial \Omega .
\end{aligned}
$$

We define the first terms in the expansion of $w_{\varepsilon}$ by $w_{0}, w_{1}$ and $C_{\varepsilon}$ analogously to $u_{0}, u_{1}$ and $B_{\varepsilon}$. Equivalently to (A 29), they satisfy

$$
\begin{aligned}
\left\|w_{\varepsilon}(x)-w_{0}(x)-\varepsilon w_{1}\left(x, \frac{x}{\varepsilon}\right)-\varepsilon C_{\varepsilon}(x)\right\|_{H^{1}(\Omega)} & \leqslant C \varepsilon\left\|w_{0}\right\|_{H^{2}(\Omega)} \\
& \leqslant C \varepsilon\|h\|_{L^{2}(\Omega)} .
\end{aligned}
$$

From (A 35), we have

$$
\left|\int_{\Omega} \varepsilon B_{\varepsilon} h\right| \leqslant\left|\int_{\Omega} a_{\varepsilon} \nabla\left(\varepsilon B_{\varepsilon}\right) \cdot \nabla\left(w_{0}+\varepsilon w_{1}+\varepsilon C_{\varepsilon}\right)\right|+C \varepsilon^{3 / 2}\left\|u_{0}\right\|_{H^{2}(\Omega)}\left\|w_{0}\right\|_{H^{2}(\Omega)} .
$$

We split the integral on the right-hand side above into three terms:

$$
I_{1}=\int_{\Omega} a_{\varepsilon} \nabla\left(\varepsilon B_{\varepsilon}\right) \cdot \nabla w_{0}=\int_{\Omega} \eta_{\varepsilon} \cdot \nabla w_{0}
$$

has the same form as the right-hand side of (A 32). Proceeding as in step 3, we see that this term can be rewritten as

$$
\begin{array}{r}
I_{1}=\varepsilon\left\{\int_{\Omega} \sum_{p=1}^{3} \tilde{\chi}\left(\frac{x}{\varepsilon}\right) \cdot\left(\nabla \rho_{p}(x) \times \nabla w_{0}\right)-\int_{\partial \Omega} \sum_{p=1}^{3}\left(\tilde{\chi}\left(\frac{x}{\varepsilon}\right) \times \nu\right) \cdot \rho_{p}(x) \nabla w_{0} \mathrm{~d} \sigma\right. \\
\left.-\int_{\partial \Omega}(\tilde{\chi} \rho(x) \times \nu) \cdot \nabla w_{0} \mathrm{~d} \sigma\right\}
\end{array}
$$

and thus, as $\tilde{\chi}(x / \varepsilon)$ is uniformly bounded in $L^{\infty}(\partial \Omega)$, as $\rho=A \nabla u_{0}$ and $\nabla w_{0}$ have at least regularity $H^{1}(\Omega)$ and $H^{1 / 2}(\partial \Omega)$, it follows that

$$
\left|I_{1}\right| \leqslant C \varepsilon\left\|u_{0}\right\|_{H^{2}(\Omega)}\left\|w_{0}\right\|_{H^{2}(\Omega)} .
$$


For the second term,

$$
\begin{aligned}
\left|I_{2}\right| & =\left|\int_{\Omega} a_{\varepsilon} \nabla\left(\varepsilon B_{\varepsilon}\right) \cdot \nabla\left(\varepsilon w_{1}\right)\right|=\left|\int_{\partial \Omega} a_{\varepsilon} \nabla\left(\varepsilon B_{\varepsilon}\right) \cdot \nu \varepsilon w_{1} \mathrm{~d} \sigma\right| \\
& \leqslant \varepsilon\left\|a_{\varepsilon} \nabla\left(\varepsilon B_{\varepsilon}\right) \cdot \nu\right\|_{H^{-1 / 2}(\partial \Omega)}\left\|\chi_{j}\left(\frac{x}{\varepsilon}\right) \frac{\partial w_{0}}{\partial x_{j}}\right\|_{H^{1 / 2}(\partial \Omega)} \cdot
\end{aligned}
$$

As $\chi_{j} \in W^{1, \infty}(Y) \cap \mathcal{C}^{1, \mu}(Y), 1 \leqslant j \leqslant 3$, one may easily check that

$$
\left\|\chi_{j}\left(\frac{x}{\varepsilon}\right) \frac{\partial w_{0}}{\partial x_{j}}\right\|_{L^{2}(\partial \Omega)} \text { and } \varepsilon\left\|\chi_{j}\left(\frac{x}{\varepsilon}\right) \frac{\partial w_{0}}{\partial x_{j}}\right\|_{H^{1}(\partial \Omega)}
$$

are uniformly bounded. By interpolation, it follows that

$$
\left\|\chi_{j}\left(\frac{x}{\varepsilon}\right) \frac{\partial w_{0}}{\partial x_{j}}\right\|_{H^{1 / 2}(\partial \Omega)} \leqslant C \varepsilon^{-1 / 2},
$$

so that, using (A 34), we obtain

$$
I_{2} \leqslant C \varepsilon\left\|u_{0}\right\|_{H^{2}(\Omega)}\left\|w_{0}\right\|_{H^{2}(\Omega)} .
$$

Finally, the third term is easily controlled using (A 35) and its equivalent for $C_{\varepsilon}$ :

$$
\begin{aligned}
\left|I_{3}\right| & =\left|\int_{\Omega} a_{\varepsilon} \nabla\left(\varepsilon B_{\varepsilon}\right) \cdot \nabla\left(\varepsilon C_{\varepsilon}\right)\right| \\
& \leqslant C\left\|\varepsilon B_{\varepsilon}\right\|_{H^{1}(\Omega)}\left\|\varepsilon C_{\varepsilon}\right\|_{H^{1}(\Omega)} \\
& \leqslant C \varepsilon\left\|u_{0}\right\|_{H^{2}(\Omega)}\left\|w_{0}\right\|_{H^{2}(\Omega)} .
\end{aligned}
$$

We thus conclude from (A 37)-(A 40) that

$$
\left|\int_{\Omega} \varepsilon B_{\varepsilon} h\right| \leqslant C \varepsilon\left\|u_{0}\right\|_{H^{2}(\Omega)}\|h\|_{L^{2}(\Omega)}
$$

and thus

$$
\left\|\varepsilon B_{\varepsilon}\right\|_{L^{2}(\Omega)} \leqslant C \varepsilon\left\|u_{0}\right\|_{H^{2}(\Omega)} .
$$

STEP 5. Finally, the uniform boundedness of $\left\|u_{1}(x, x / \varepsilon)\right\|_{L^{2}(\Omega)}$, (A 29) and (A 41) yield

$$
\begin{aligned}
\left\|u_{\varepsilon}(x)-u_{0}(x)\right\|_{L^{2}(\Omega)} \leqslant & \left\|u_{\varepsilon}(x)-u_{0}(x)-\varepsilon u_{1}\left(x, \frac{x}{\varepsilon}\right)-\varepsilon B_{\varepsilon}(x)\right\|_{H^{1}(\Omega)} \\
& +\varepsilon\left\|u_{1}\left(x, \frac{x}{\varepsilon}\right)\right\|_{L^{2}(\Omega)}+\left\|\varepsilon B_{\varepsilon}(x)\right\|_{L^{2}(\Omega)} \\
\leqslant & \leqslant C \varepsilon\left\|u_{0}\right\|_{H^{2}(\Omega)} .
\end{aligned}
$$

\section{A.3. Proof of theorem 3.6}

Let $\chi$ and $\Phi$ be the first and the second-order matrix of correctors, solutions of the cell problems (3.4), and (3.5), (3.6), respectively. 
Consider the auxiliary function

$$
z_{\varepsilon}=u_{\varepsilon}-u_{0}-\varepsilon \chi\left(\frac{x}{\varepsilon}\right) \cdot \nabla u_{0}-\varepsilon^{2} \Phi\left(\frac{x}{\varepsilon}\right): \nabla^{2} u_{0}
$$

Applying $L_{\varepsilon}$ to $z_{\varepsilon}$, we obtain (with $\chi_{\varepsilon}(x)=\chi(x / \varepsilon)$ and $\Phi_{\varepsilon}(x)=\Phi(x / \varepsilon)$ )

$$
\begin{aligned}
L_{\varepsilon} z_{\varepsilon}= & \operatorname{div}\left(\left(a_{\varepsilon} I-A\right) \nabla u_{0}\right)+\varepsilon \operatorname{div}\left(a_{\varepsilon} \nabla\left(\chi_{\varepsilon} \nabla u_{0}\right)\right)+\varepsilon^{2} \operatorname{div}\left(a_{\varepsilon} \nabla\left(\Phi_{\varepsilon} \nabla^{2} u_{0}\right)\right) \\
= & \operatorname{div}\left(\left(a_{\varepsilon}\left(I+\nabla_{y} \chi\right)-A\right) \nabla u_{0}\right)+\varepsilon \operatorname{div}\left(a_{\varepsilon} \chi_{\varepsilon} \nabla^{2} u_{0}\right) \\
& \quad+\varepsilon \operatorname{div}\left(a_{\varepsilon} \nabla_{y} \Phi \nabla^{2} u_{0}\right)+\varepsilon^{2} \operatorname{div}\left(a_{\varepsilon} \Phi_{\varepsilon} \nabla^{3} u_{0}\right) \\
= & \left(a\left(I+\nabla_{y} \chi\right)-A\right) \nabla^{2} u_{0}+\varepsilon a \chi_{\varepsilon} \nabla^{3} u_{0}+\operatorname{div}_{y}(a \chi) \nabla^{2} u_{0} \\
& \quad+\operatorname{div}_{y}\left(a \nabla_{y} \Phi\right) \nabla^{2} u_{0}+\varepsilon a \nabla_{y} \Phi \nabla^{3} u_{0}+\varepsilon^{2} \operatorname{div}\left(a_{\varepsilon} \Phi_{\varepsilon} \nabla^{3} u_{0}\right) .
\end{aligned}
$$

Since $\Phi$ is the solution of $(3.5),(3.6)$ and $\int_{Y} B(y) \mathrm{d} y=A(y)$, we get

$$
L_{\varepsilon} z_{\varepsilon}=\varepsilon\left[F_{\varepsilon}+\varepsilon \operatorname{div}\left(b_{\varepsilon} H_{\varepsilon}\right)\right]
$$

with

$$
\begin{aligned}
F_{\varepsilon}(x) & =a\left(\frac{x}{\varepsilon}\right) \chi\left(\frac{x}{\varepsilon}\right) \nabla^{3} u_{0}(x)+a\left(\frac{x}{\varepsilon}\right) \nabla_{y} \Phi\left(\frac{x}{\varepsilon}\right) \nabla^{3} u_{0}(x), \\
b(y) & =a(y) \Phi(y), \\
H_{\varepsilon}(x) & =\nabla^{3} u_{0}(x) .
\end{aligned}
$$

Noting that $\Phi$ has regularity $\mathcal{C}^{0, \mu}$ in $\bar{D}_{0}$ and in $\overline{Y \backslash D_{0}}$ by $(3.5)$ and (3.13), we can apply theorem 3.4: for a suitable constant $C$,

$$
\left\|F_{\varepsilon}\right\|_{L^{\infty}(\Omega)}+\left\|H_{\varepsilon}\right\|_{\mathcal{C}^{0, \mu}(\Omega)} \leqslant C .
$$

The interior gradient estimates (3.15) applied to $z_{\varepsilon}$ and lemma 3.5 then show that

$$
\left\|z_{\varepsilon}\right\|_{L^{\infty}(\omega)}+\left\|\nabla z_{\varepsilon}\right\|_{L^{\infty}(\omega)} \leqslant\left(\left\|z_{\varepsilon}\right\|_{L^{2}(\Omega)}+C \varepsilon\right) .
$$

Since by hypothesis $\left\|u_{\varepsilon}-u_{0}\right\|_{L^{2}(\Omega)} \leqslant C \varepsilon^{\sigma}$, we conclude that

$$
\left|u_{\varepsilon}(x)-u_{0}(x)\right|+\left|\nabla u_{\varepsilon}(x)-\left(I+\nabla_{y} \chi\left(\frac{x}{\varepsilon}\right)\right) \nabla u_{0}(x)\right| \leqslant C \varepsilon^{\sigma} \quad \text { a.e. } x \in \omega .
$$

\section{A.4. Proof of theorem 3.7}

From the results of $\S 2$, we see that $G_{\varepsilon}$ is Hölder continuous away from its singular point and that there exists a constant $C$ that depends only on $\lambda$ such that

$$
\left\|G_{\varepsilon}(\cdot, y)\right\|_{L^{2}(\Omega)} \leqslant C \quad \text { for } y \in \Omega,
$$

since $L^{2}(\Omega) \subset L_{n / n-2}^{*}(\Omega)$ when $n=3$.

Let $\omega_{1}$ and $\omega_{2}$ be smooth domains such that $\omega \subset \subset \omega_{1} \subset \subset \omega_{2} \subset \subset \Omega$ and let $y \in \Omega \backslash \omega_{2}$.

Let

$$
\begin{aligned}
\sigma_{\varepsilon} & =\sup \left\{\left|G_{\varepsilon}(x, y)-G_{0}(x, y)\right|: x \in \bar{\omega}_{1}\right\} \\
& =:\left|G_{\varepsilon}\left(x_{\varepsilon}, y\right)-G_{0}\left(x_{\varepsilon}, y\right)\right| .
\end{aligned}
$$


As $L_{\varepsilon} G_{\varepsilon}(\cdot, y)=0$ in $\omega_{2}$, theorem 3.4 shows that there is a positive constant $C$ independent of $\varepsilon$ and $y$, such that

$$
\left\|\nabla G_{\varepsilon}(\cdot, y)\right\|_{L^{\infty}\left(\omega_{2}\right)}+\left\|\nabla G_{0}(\cdot, y)\right\|_{L^{\infty}\left(\omega_{2}\right)} \leqslant C .
$$

Setting $\rho=\min \left(\sigma_{\varepsilon} / 2 C\right.$, $\left.\operatorname{dist}\left(\omega_{1}, \omega_{2}\right)\right)$, we see that

$$
\left|G_{\varepsilon}(x, y)-G_{0}(x, y)\right| \geqslant \frac{1}{2} \sigma_{\varepsilon} \quad \text { for } x \in B_{\rho}\left(x_{\varepsilon}\right) \cap \omega_{1} .
$$

In particular, $G_{\varepsilon}(\cdot, y)-G_{0}(\cdot, y)$ keeps constant sign in $B_{\rho}\left(x_{\varepsilon}\right)$. Let $f \in \mathcal{C}_{0}^{\infty}\left(B_{\rho}\left(x_{\varepsilon}\right)\right)$, such that $0 \leqslant f \leqslant 1$ and $f \equiv 1$ on $B_{\rho / 2}\left(x_{\varepsilon}\right)$. We consider the solutions $w_{\varepsilon}$ and $w_{0}$, vanishing on $\partial \Omega$, of

$$
L_{\varepsilon} w_{\varepsilon}=f \quad \text { and } \quad L_{0} w_{0}=f \quad \text { in } \Omega .
$$

Theorem 2.3 in [1] gives us an interior $L^{2}$ estimate for the convergence rate of $w_{\varepsilon}$ to $w_{0}$ : for some constant $C$ independent of $\varepsilon$,

$$
\left\|w_{\varepsilon}-w_{0}\right\|_{L^{2}\left(\omega_{2}\right)} \leqslant C \varepsilon .
$$

Applying theorem 3.6, we see that

$$
w_{0} \|_{L^{\infty}\left(\omega_{1}\right)} \leqslant C \varepsilon .
$$

Thus, for $x \in B_{\rho / 2}\left(x_{\varepsilon}\right) \cap \omega_{1}$, we obtain

$$
\begin{aligned}
C \varepsilon & \geqslant\left|w_{\varepsilon}(x)-w_{0}(x)\right| \\
& =\int_{B_{\rho}\left(x_{\varepsilon}\right)}\left|G_{\varepsilon}(x, y)-G_{0}(x, y)\right| f(y) \mathrm{d} y . \\
& \geqslant \int_{B_{\rho / 2}\left(x_{\varepsilon}\right) \cap \omega_{1}} \frac{1}{2} \sigma_{\varepsilon} \mathrm{d} y .
\end{aligned}
$$

As the Lebesgue measure of $B_{\rho / 2}\left(x_{\varepsilon}\right) \cap \omega_{1}$ is proportional to $\rho^{3}$, we conclude from its definition that $\sigma_{\varepsilon}=O\left(\varepsilon^{1 / 4}\right)$ and that

$$
\left\|G_{\varepsilon}(\cdot, y)-G_{0}(\cdot, y)\right\|_{L^{\infty}\left(\omega_{1}\right)} \leqslant C \varepsilon^{1 / 4} .
$$

Moreover, as this estimate also holds in $L^{2}\left(\omega_{1}\right)$, another application of theorem 3.6 yields (3.20), (3.21).

\section{References}

1 G. Allaire and M. Amar. Boundary layer tails in periodic homogenization. ESAIM: Control Optim. Calc. Variations 4 (1999), 209-243.

2 H. Ammari and H. Kang. Reconstruction of small inhomogeneities from boundary measurements. Lecture Notes in Mathematics, vol. 1846 (Springer, 2004).

3 H. Ammari, E. Iakovleva and D. Lesselier. A MusIC algorithm for locating small inclusions buried in a half-space from the scattering amplitude at a fixed frequency. Multiscale Model. Simul. 3 (2005), 597-628.

4 M. Avellaneda and F. H. Lin. Compactness methods in the theory of homogenization. Commun. Pure Appl. Math. 40 (1987), 803-847.

$5 \quad$ M. Avellaneda and F. H. Lin. $L^{p}$ bounds on singular integrals in homogenization. Commun. Pure Appl. Math. 44 (1991), 897-910. 
6 A. Bensoussan, J. L. Lions and G. Papanicolaou. Asymptotic analysis of periodic structures (Amsterdam: North-Holland, 1978).

7 M. Brühl, M. Hancke and M. Vogelius. A direct impedance tomography algorithm for locating small inhomogeneities. Numer. Math. 93 (2003), 635-654.

8 S. Campanato. Proprietà di Hölderianita di alcune classi di funzioni. Annli Scuola Norm. Sup. Pisa 17 (1963), 175-188.

$9 \quad$ Y. Capdeboscq and M. Vogelius. A general representation formula for boundary voltage perturbations caused by internal conductivity inhomogeneities of low volume fraction. Math. Model. Numer. Analysis 37 (2003), 159-173.

10 D. J. Fengya, S. Moskow and M. Vogelius. Identification of conductivity imperfections of small diameter by boundary measurements: continuous dependence and computational reconstruction. Inv. Probl. 14 (1998), 553-595.

11 M. Giaquinta. Multiple integrals in the calculus of variations and nonlinear elliptic systems. Annals of Mathematics Studies, vol. 105 (Princeton University Press, 1983).

12 M. Grüter and K. O. Widman. The Green function for uniformly elliptic equations. Manuscr. Math. 37 (1982), 303-342.

13 J. D. Joannopoulos, R. D. Meade and J. N. Winn. Photonic crystals: molding the flow of light (Princeton University Press, 1995).

14 P. Kuchment. The mathematics of photonic crystals. In Mathematical modeling in optical science (ed. G. Bao, L. Cowsar and W. Masters). Frontiers in Applied Mathematics, vol. 22, ch. 7, pp. 207-272 (Philadelphia, PA: SIAM, 2001).

15 Y. Y. Li and L. Nirenberg. Estimates for elliptic systems from composite material. Commun. Pure Appl. Math. 56 (2003), 892-925.

16 Y. Y. Li and M. Vogelius. Gradient estimates for solutions of divergence form elliptic equations with discontinuous coefficients. Arch. Ration. Mech. Analysis 153 (2000), 91151.

17 J.-L. Lions and E. Magenes. Problèmes aux limites non homogènes et applications, vol. 1 (Dunod, 1968).

18 W. Littman, G. Stampacchia and H. F. Weinberger. Regular points for elliptic equations with discontinuous coefficients. Annli Scuola Norm. Sup. Pisa 17 (1963), 43-77.

19 S. Moskow and M. Vogelius. First-order corrections to the homogenized eigenvalues of a periodic composite medium: a convergence proof. Proc. R. Soc. Edinb. A 127 (1997), 12631299.

20 S. Moskow and M. Vogelius. First-order corrections to the homogenized eigenvalues of a periodic composite medium: the case of Neumann boundary conditions. (Submitted.)

21 G. Stampacchia. Contributi alla regolarizzazione delle soluzioni dei problemi al contorno per equazioni del secondo ordine ellittiche. Annli Scuola Norm. Sup. Pisa 12 (1958), 223-245.

22 G. Stampacchia. Problemi al contorno ellittici, con dati discontinui, dotati di soluzioni Hölderiane. Annli Mat. 51 (1960), 1-38.

23 V. V. Zhikov, S. M. Kozlov, O. A. Oleinik and K. T. Ngoan. Averaging and G-convergence of differential operators. Usp. Mat. Nauk 34 (1979), 65-128. (Engl. transl. in Russ. Math. Surv. 34 (1979), 69-147.)

(Issued 11 August 2006) 